\title{
A spatially adaptive nonparametric regression image deblurring
}

Vladimir Katkovnik, Karen Egiazarian, Alessandro Foi, Jaakko Astola

Signal Processing Laboratory, Tampere University of Technology, Finland,

katkov, karen, foi, jta@cs.tut.fi 


\section{Introduction}

We wish to recover a $2 D$ image intensity $y(x)$, from observations

$$
z(x)=(y \star v)(x)+\eta(x),
$$

where $v$ is the blurring point spread function $(P S F)$ of a linear discrete convolution, $x \in X$,

$$
X=\left\{\left(k_{1}, k_{2}\right), k_{1}=1,2, \ldots, n_{1}, k_{2}=1,2, \ldots, n_{2}\right\}
$$

and $\eta$ is i.i.d. Gaussian noise with the variance $\sigma^{2}$.

The $v(x)$ is known.

The blurring phenomenon, modelled by the kernel $v$ (continuous or discrete), is very evident in many image applications.

Inverse problems arise in optical systems, satellite imaging, radiometry, ultrasonic and magnetic resonance imaging, etc. 
In the $2 D$ discrete Fourier transform $(D F T)$ domain we have

$$
Z(f)=Y(f) V(f)+\eta(f),
$$

with $Z(f), Y(f), V(f)$ and $\eta(f)$ of the respective size $n_{1} \times n_{2}$ being the $D F T$ of the corresponding variables and the $2 D$ normalized frequency $f \in F$,

$$
\begin{aligned}
& F=\left\{\left(f_{1}, f_{2}\right), f_{1}=2 \pi k_{1} / n_{1}, f_{2}=2 \pi k_{2} / n_{2},\right. \\
& \left.k_{1}=0,1, \ldots, n_{1}-1, k_{2}=0,1, \ldots, n_{2}-1\right\} .
\end{aligned}
$$

An unbiased estimate of $Y(f)$ can be obtained as a straightforward pure ('naive') inverse solution

$$
\hat{Y}(f)=\frac{1}{V(f)} Z(f) .
$$

In the frequency domain being ill-posed means that $V(f)$ may take zero or close to zero values. 
A regularized inverse operator gives instead of the pure inverse

$$
\hat{Y}(f)=\frac{V(-f)}{|V(f)|^{2}+\varepsilon^{2}} Z(f),
$$

where $\varepsilon>0$ is a regularization parameter.

This typically produces a reconstruction in which certain features of the original image are 'smoothed away' and it seems to be a blurred version of the original.

It would be of interest to obtain sharper reconstructions for objects with edges.

The phenomenon of blurring and the goal of edge recovery have been studied by many researchers over the last few years. 
A common point of most methods is that some basis functions are applied for a approximation of the object function $y(x)$ in the form of series with coefficients defined from the observations.

These functions may be Fourier harmonics, eigenfunctions of the convolution operator in SVD methods or wavelets in wavelet based decompositions.

\section{LPA estimation}

Basically different ideas and methods arise from a nonparametric regression approach. It is assumed that the function $y(x)$ is well approximated by a polynomial in some neighborhood of the point of interest $x$.

The coefficients of the polynomial fit are found by the weighted least square method. This approximation is used in order to calculate an estimate for the point of interest $x$ called also the "centre" of the $L P A$. 
This pointwise procedure determines a nonparametric character of the LPA estimation.

Let $x$ be a "center" (reference point) of the LPA. The estimate for the point $x_{s}$ in the neighborhood of the center $x$ is presented as an expansion:

$$
\begin{aligned}
& \tilde{y}\left(x, x_{s}\right)=C^{T} \phi_{h}\left(x-x_{s}\right), \phi_{h}(x)=\phi(x / h) \\
& \phi(x)=\left(\phi_{1}(x), \phi_{2}(x), \ldots, \phi_{M}(x)\right)^{T}, \\
& C=\left(C_{1}, C_{2}, \ldots, C_{M}\right)^{T},
\end{aligned}
$$

where $\phi(x) \in R^{M}$ is a vector of linear independent $2 D$ polynomials of the powers from 0 up to $m, C \in R^{M}$ is a vector of parameters of this model. 
The conventional quadratic criteria function can be applied in order find $C$ :

$$
J_{h}(x)=\sum_{x_{s}} w_{h}\left(x-x_{s}\right)\left(z\left(x_{s}\right)-\tilde{y}\left(x, x_{s}\right)\right)^{2}, w_{h}(x)=w(x / h) / h^{2},
$$

where the window $w$ formalizes the localized fitting in a neighborhood of the centre $x$.

The scale parameter $h>0$ determines the "size" of the neighborhood.

The LPA estimate of $y(x)$ is defined as

$$
\hat{y}_{h}(x)=\left.\tilde{y}\left(x, x_{s}\right)\right|_{x_{s}=x}=C^{T}(x) \phi_{h}(0),
$$

and used for estimate calculation for $x_{s}=x$ only.

It is a key idea of the pointwise nonparametric estimate design. 
Thus, we arrive to a linear discrete kernel estimator defined on the lattice $X$ and given by the kernel $g_{h}(x), x \in X \subset R^{2}$, with the scale (window size) parameter $h>0$ :

$$
y_{h}(x)=\left(g_{h} \circledast y\right)(x)
$$

The kernel $g_{h}(x)$ is defined by the equations

$$
\begin{aligned}
& g_{h}(x)=w_{h}(x) \phi_{h}^{T}(0) \Phi_{h}^{-1} \phi_{h}(x), \\
& \Phi_{h}=\sum w_{h}(x) \phi_{h}(x) \phi_{h}^{T}(x) .
\end{aligned}
$$


The following holds for $g_{h}$ :

(G1) The polynomial smoothness, $m$ vanishing moments,

$$
\left(g_{h} \circledast x^{k}\right)(0)=\delta_{|k|, 0},|k| \leq m,
$$

where $k=\left(k_{1}, k_{2}\right)$ is a multi-index,

$$
|k|=k_{1}+k_{2}, x^{k}=x_{1}^{k_{1}} x_{2}^{k_{2}} ;
$$

(G2)

$$
\left\|g_{h}\right\|^{2}=\sum_{x}\left|g_{h}(x)\right|^{2} \leq B h^{-b}, B, b>0 .
$$

Then, we say that the $g_{h}$ is a smoothing kernel estimator of the order $m$. 


\section{Idea of the deblurring algorithm}

The smoothed image intensity $y_{h}(x)$ is used instead of the original $y(x)$ as a solution of the inverse problem and the scale parameter $h$ is exploited in order to suppress noise as much as possible while preserving details of the object function $y(x)$.

Applying the kernel operator $g_{h}$ to the both sides of the observation equation we yield

$$
z_{h}(x)=g_{h}(\circledast(y \circledast v))(x)+\eta_{h}(x)=\left(y_{h} \circledast v\right)(x)+\eta_{h}(x),
$$

where $y_{h}(x)=\left(g_{h} \circledast y\right)(x)$.

In the frequency domain this equation can be represented as:

$$
Z_{h}(f)=Y_{h}(f) V(f)+\eta_{h}(f),
$$

where $Z_{h}(f), Y_{h}(f)$, and $\eta_{h}(f)$ stand for DFT of the corresponding smoothed functions. 
The following three types of "solutions" can be used:

(A) Pure (naive) inverse $(P I)$

$$
\hat{Y}_{h}(f)=\frac{1}{V(f)} Z_{h}(f)=\frac{G_{h}(f)}{V(f)} Z(f), V(f) \neq 0, f \in F,
$$

(B) Regularized inverse (RI)

$$
\hat{Y}_{h}(f)=\frac{V(-f)}{|V(f)|^{2}+\varepsilon^{2}} Z_{h}(f)=\frac{V(-f) G_{h}(f)}{|V(f)|^{2}+\varepsilon^{2}} Z(f),
$$

(C) Regularized Wiener inverse $(R W I)$

$$
\hat{Y}_{h}(f)=\frac{V(-f)\left|Y_{h}(f)\right|^{2}}{\left|V(f) Y_{h}(f)\right|^{2}+\varepsilon^{2} \sigma^{2}\left|G_{h}(f)\right|^{2}} Z_{h}(f)=\frac{V(-f)|Y(f)|^{2} G_{h}(f)}{|V(f) Y(f)|^{2}+\varepsilon^{2} \sigma^{2}} Z(f),
$$

where $\varepsilon$ is a regularization parameter. 


\section{Pointwise spatially adaptive deblurring : ICI rule}

The parameter $h$ should be selected in such way that the noise in $\hat{y}_{h}(x)$ will be suppressed as much as possible provided that the specific features of the object $y(x)$ are preserved in $\hat{y}_{h}(x)$.

Consider a finite set of scale parameters $h$ :

$$
H=\left\{h_{1}<h_{2}<\ldots<h_{J}\right\},
$$

starting with a quite small $h_{1}$, and determine a sequence of the confidence intervals $D(j)$ of the biased estimates obtained with the windows $h=h_{j}$ as follows

$$
D(j)=\left[\hat{y}_{h_{j}}(x)-\Gamma \cdot \sigma_{\hat{y}_{h_{j}}}, \hat{y}_{h_{j}}(x)+\Gamma \cdot \sigma_{\hat{y}_{h_{j}}}\right] \text {, }
$$

where $\sigma_{\hat{y}_{h}}^{2}$ is the variance of the estimate $\hat{y}_{h_{j}}(x)$ and $\Gamma$ is a threshold of the confidence interval. 
The following ICI rule (statistic) is used in order to obtain the adaptive window size (Goldenshluger-Nemirovski $(1994,1997)$ ):

Consider the intersection of the intervals $D(j), 1 \leq j \leq i$, with increasing $i$, and let $i^{+}$be the largest of those $i$ for which the intervals $D(j), 1 \leq j \leq i$, have a point in common.

This $i^{+}$defines the adaptive window size and the adaptive LPA estimate as follows

$$
\hat{y}^{+}(x)=\hat{y}_{h^{+}(x)}(x), h^{+}(x)=h_{i^{+}} .
$$




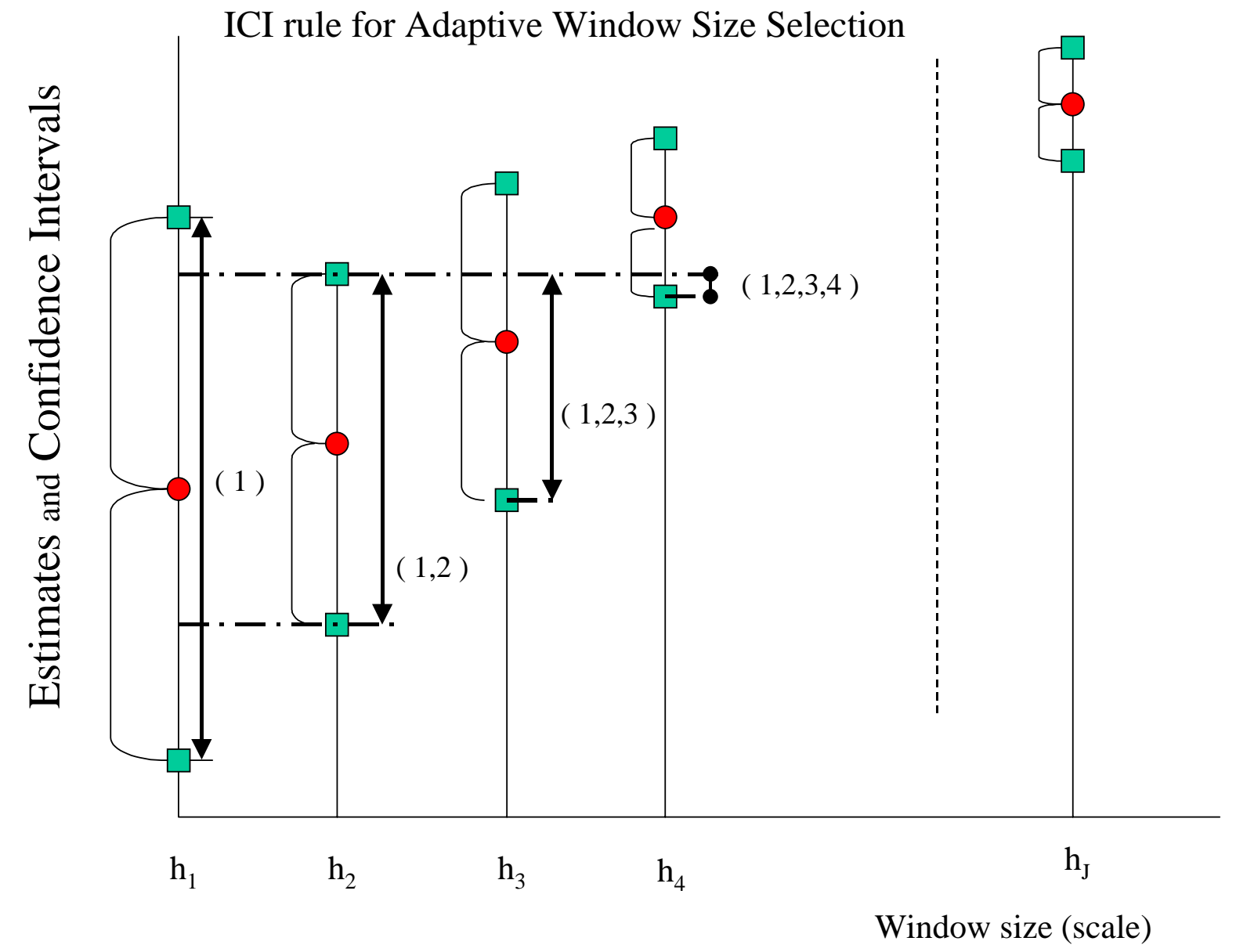


This window size ICI selection procedure requires knowledge of the estimate and its variance only.

It is equally applicable to all three algorithms $P I, R I, R W I$ with the their variances defined respectively by the formulas:

$$
\begin{aligned}
\sigma_{\hat{y}_{h}}^{2} & =\frac{\sigma^{2}}{n_{1} n_{2}}\left\|\frac{G_{h}(f)}{V(f)}\right\|_{2}^{2}, \\
\sigma_{\hat{y}_{h}}^{2} & =\frac{\sigma^{2}}{n_{1} n_{2}}\left\|\frac{V(-f) G_{h}(f)}{|V(f)|^{2}+\varepsilon^{2}}\right\|_{2}^{2}, \\
\sigma_{\hat{y}_{h}}^{2} & =\frac{\sigma^{2}}{n_{1} n_{2}}\left\|\frac{V(-f)|Y(f)|^{2} G_{h}(f)}{|V(f) Y(f)|^{2}+\varepsilon^{2} \sigma^{2}}\right\|_{2}^{2} .
\end{aligned}
$$

The kernel operator $g_{h}$ should be agreed with the PSF convolution kernel $v$, i.e. the above variances are assumed to be finite. 


\section{Implementation}

Modification of the basic ideas.

(1) Two step algorithm is developed:

(a) The RI deconvolution procedure gives the image estimate used as a reference signal on the second step;

(b) The RWI deconvolution gives the final estimate.

Both steps use the ICI rule for the adaptive scale selection.

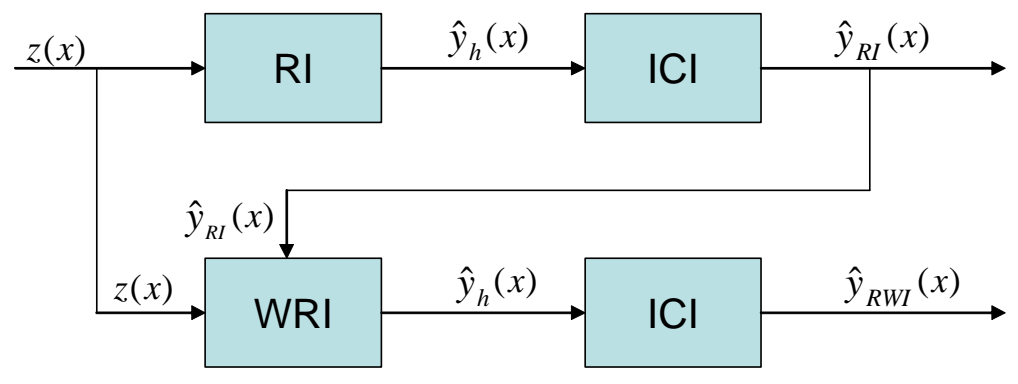




\section{(2) Directional LPA}

A symmetric window-weight $w$ in the LPA is a good choice if $y(x)$ is isotropic in a neighborhood of an estimation point. However if $y(x)$ is anisotropic nonsymmetric approximations of $y(x)$ become much more reasonable. To deal with the anisotropy of $y(x)$ multiple directional LPA kernels and estimates are exploited.

The neighborhood of the pixel $x$ is separated in a number overlapping or nonoverlapping subareas and the narrow directional kernels are obtained by rotation of $g_{h}$ :

$$
\begin{aligned}
& g_{h}(k, \theta)=w_{h}(U k) \phi_{h}^{T}(0) \Phi_{h}^{-1} \phi_{h}(U k), \Phi_{h}=\sum_{k} w_{h}(U k) \phi_{h}(U k) \phi_{h}^{T}(U k), \\
& U=\left(\begin{array}{c}
\cos \theta \sin \theta \\
-\sin \theta \cos \theta
\end{array}\right) .
\end{aligned}
$$




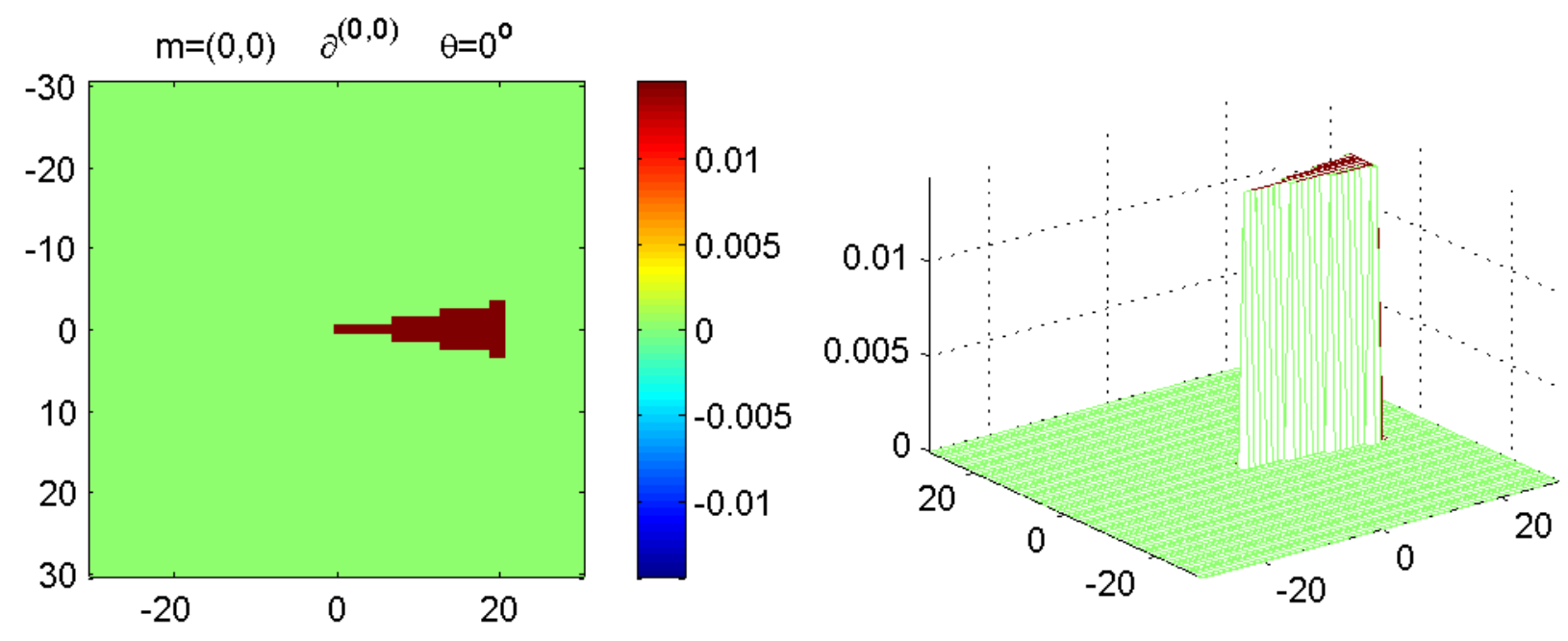

|FT|

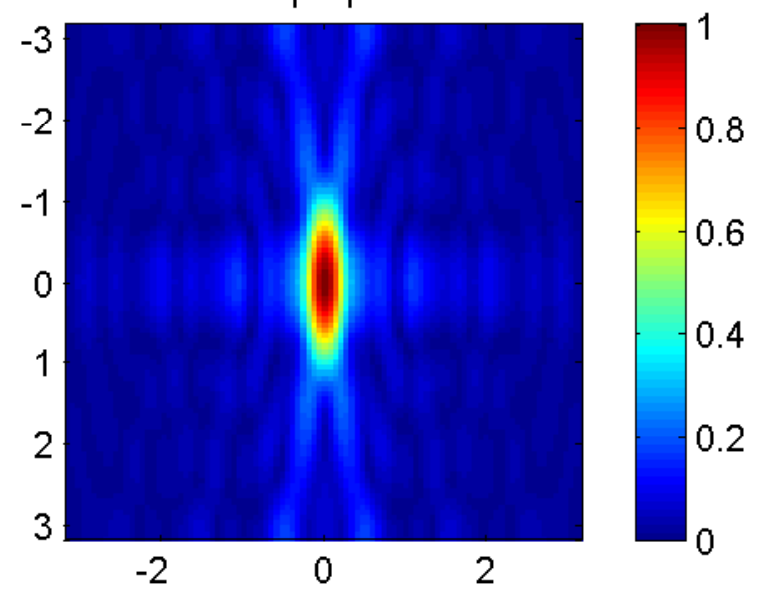

|FT|

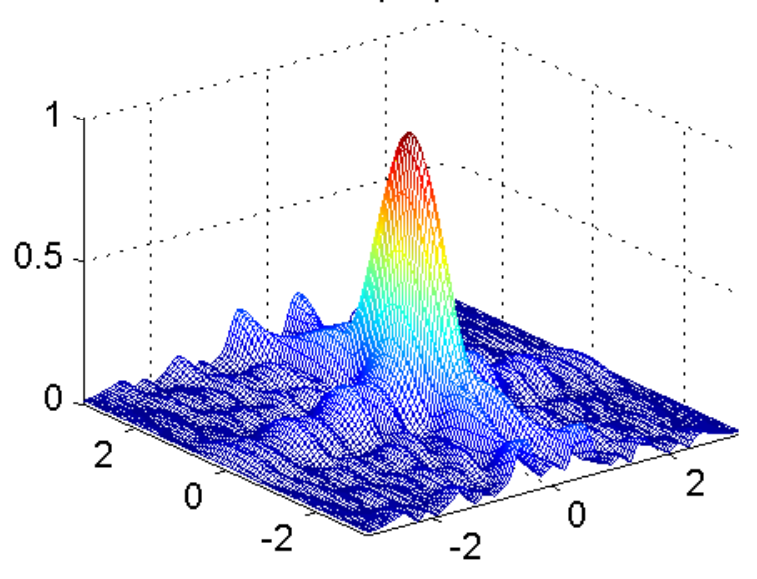




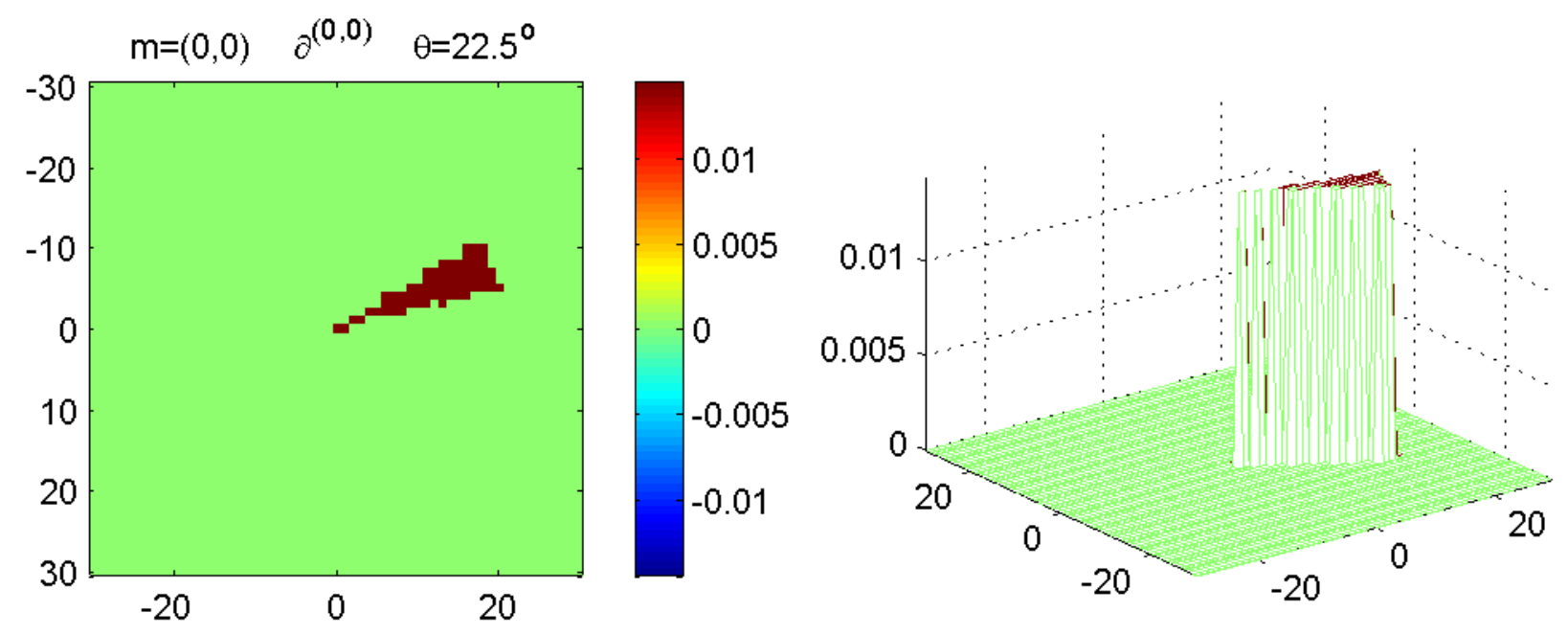

|FT|

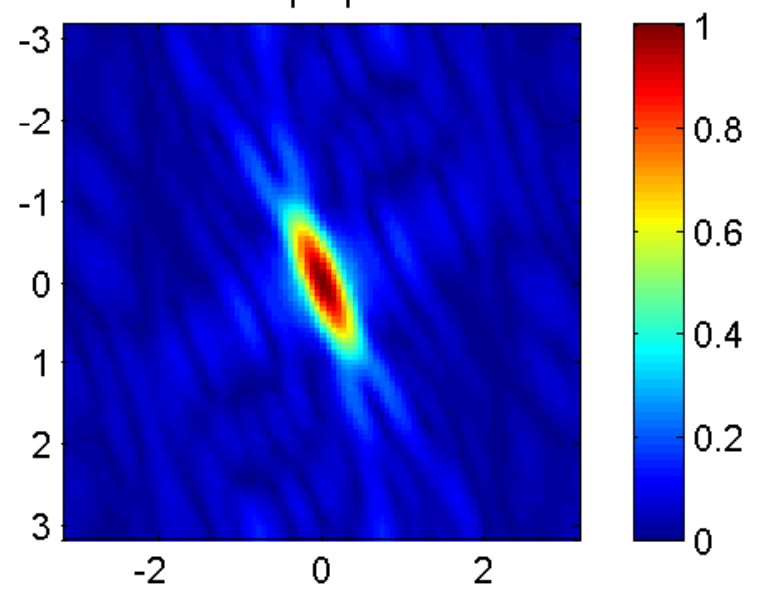

|FT|

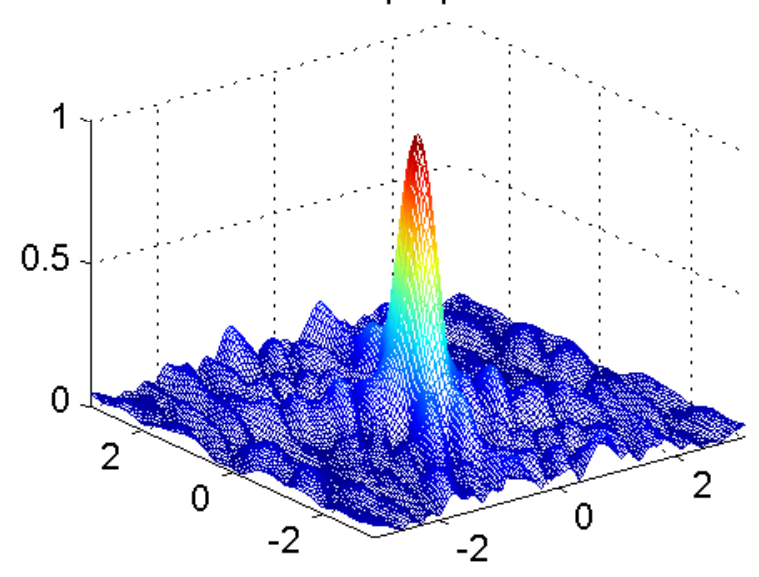




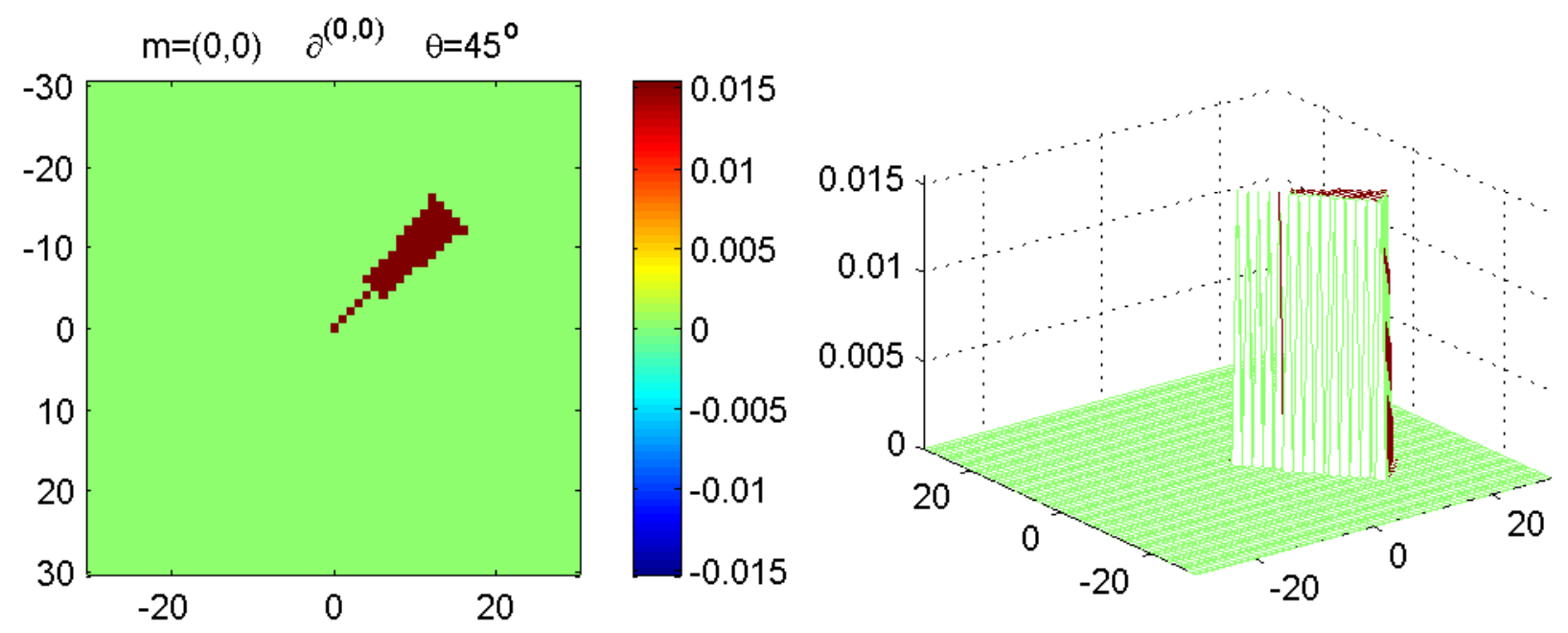

|FT|
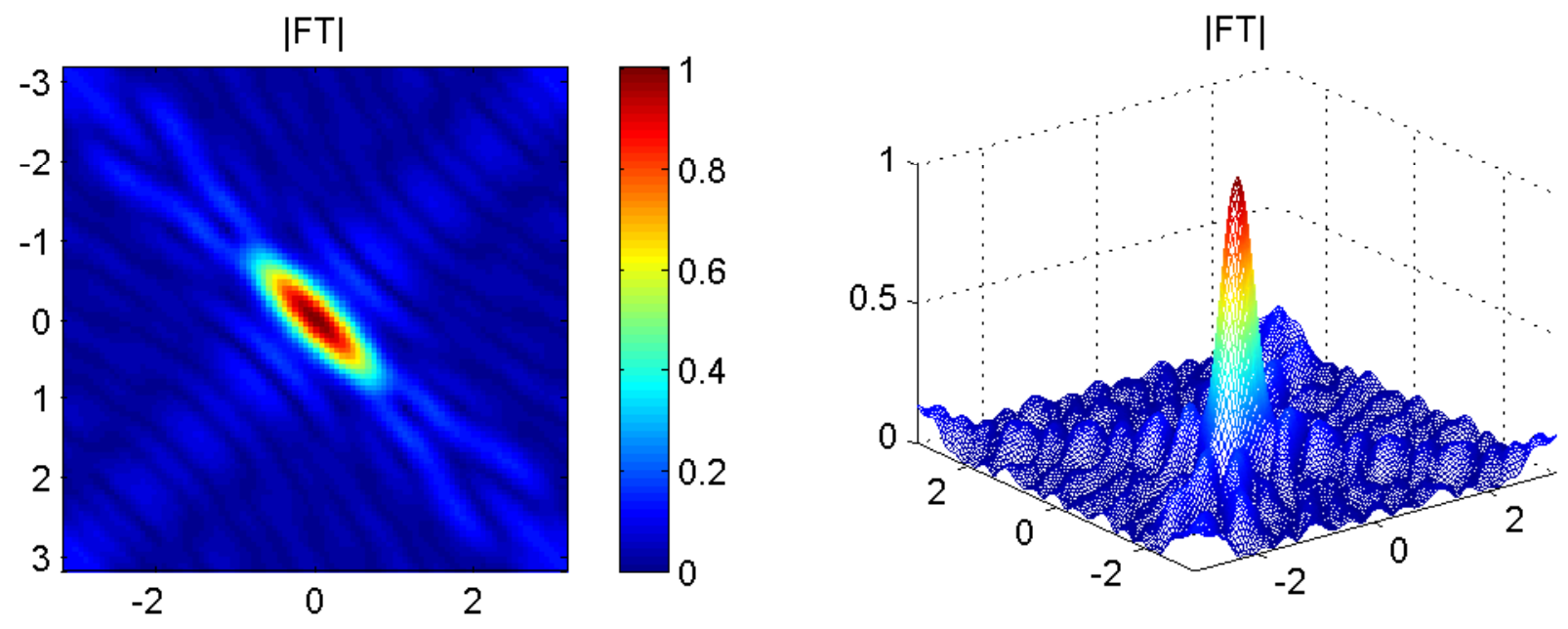


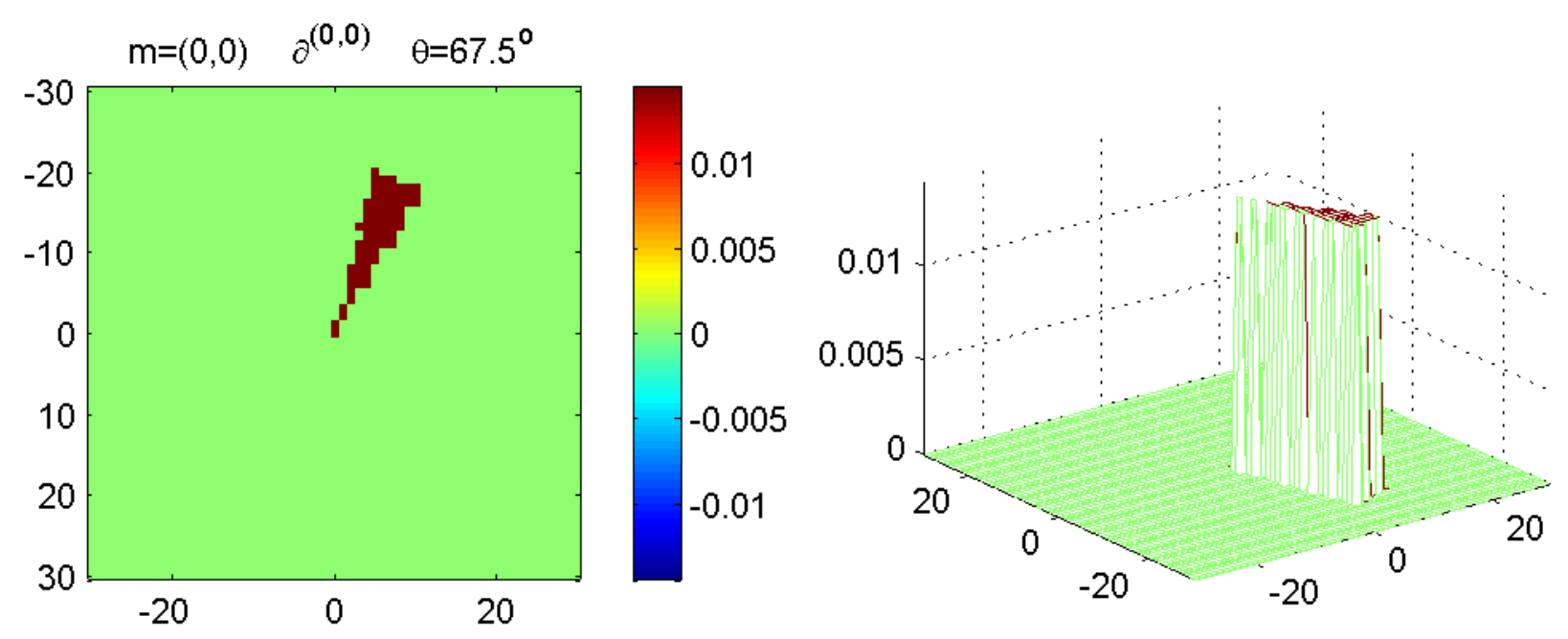

|FT|
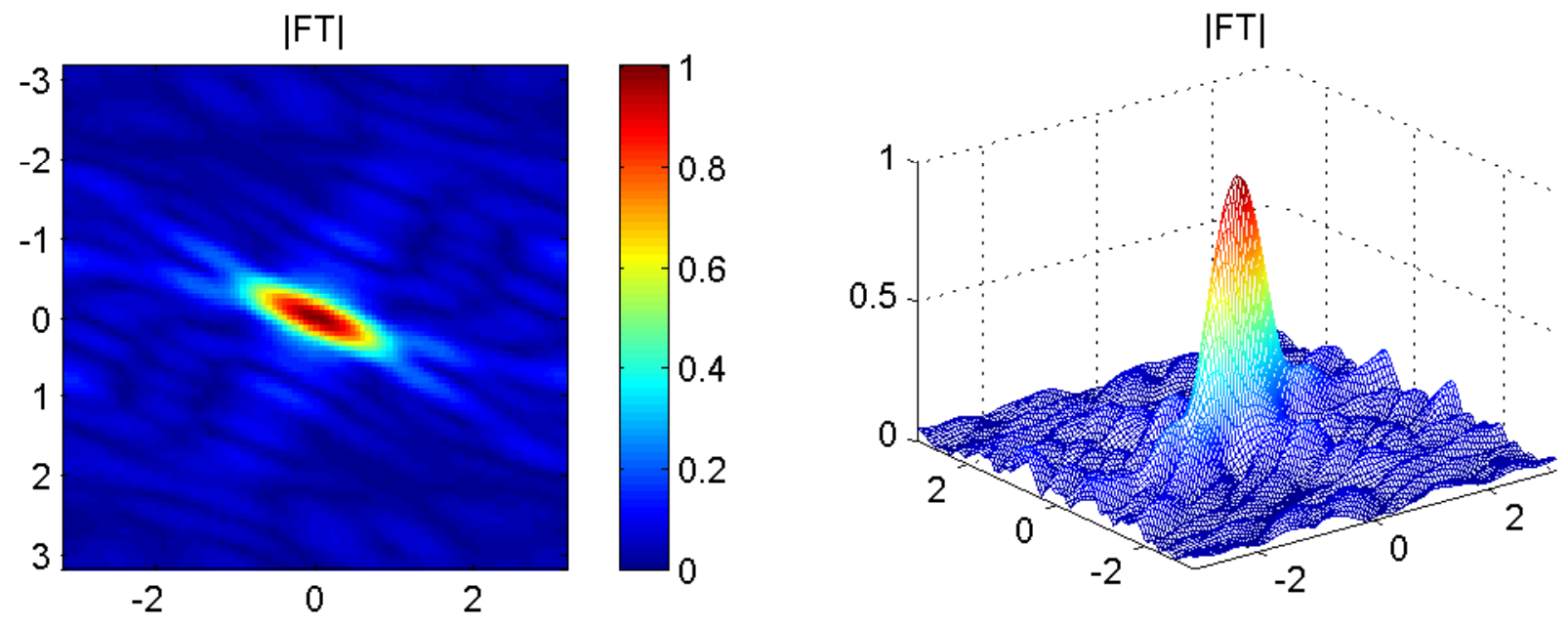


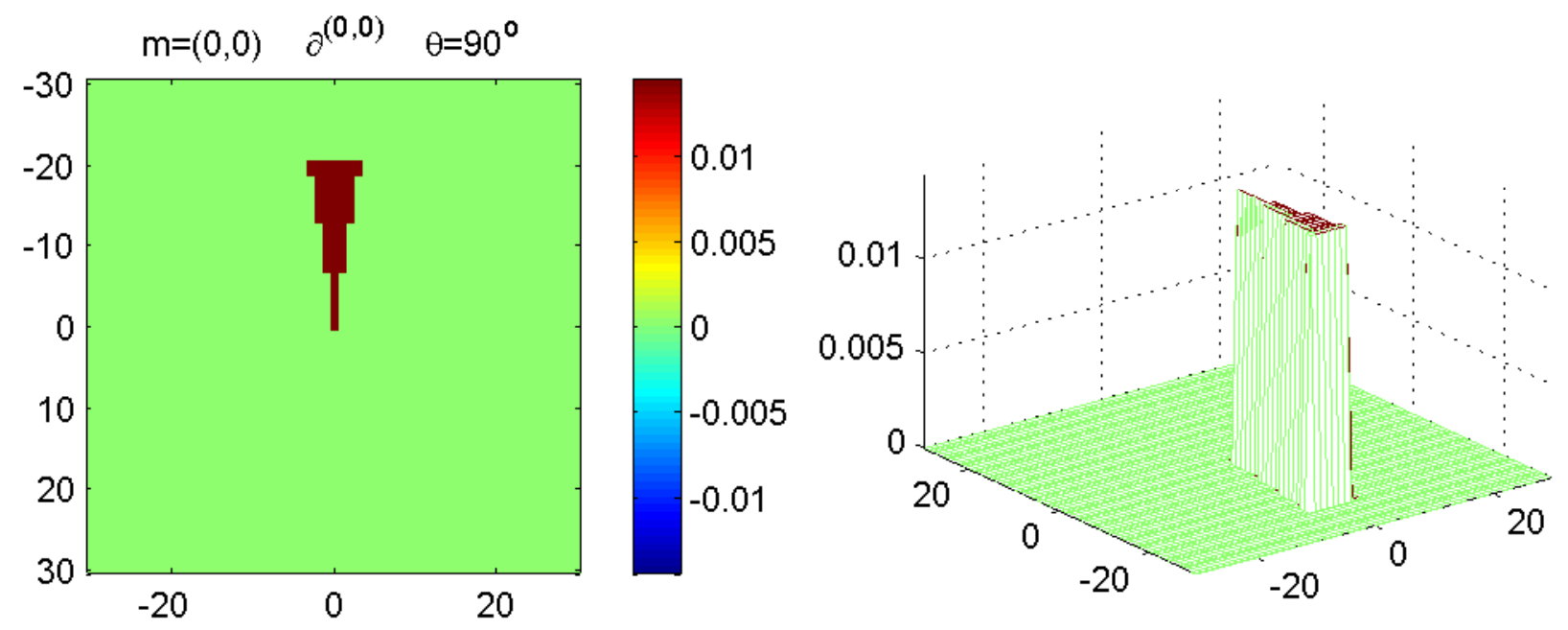

|FT|
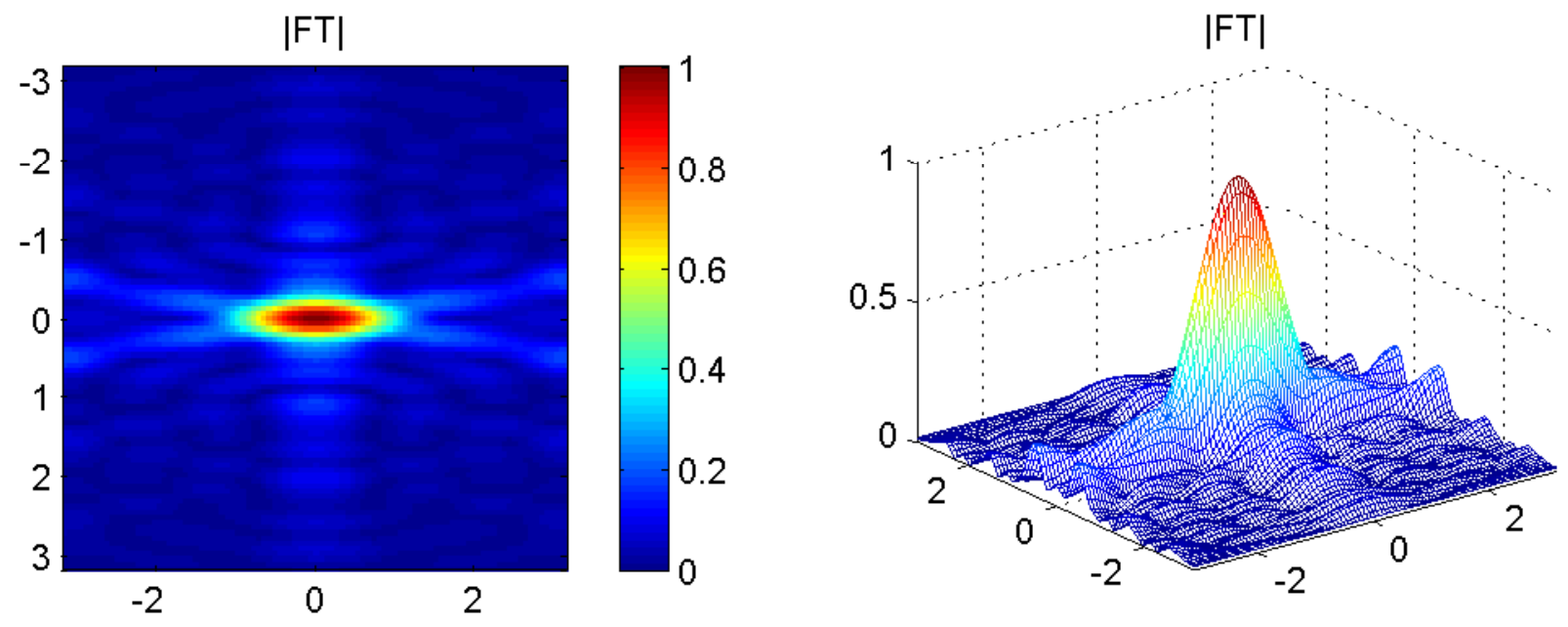

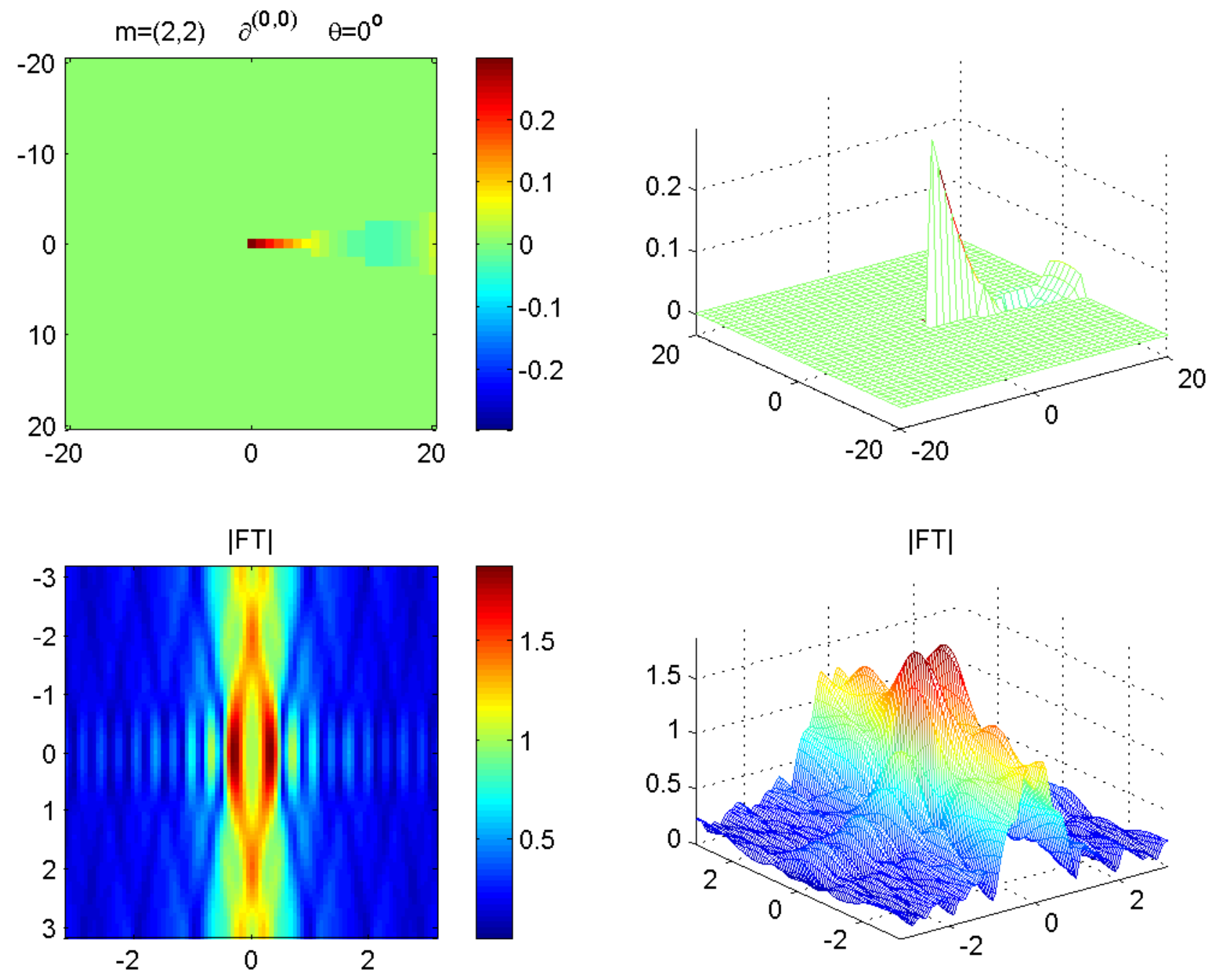

|FT|

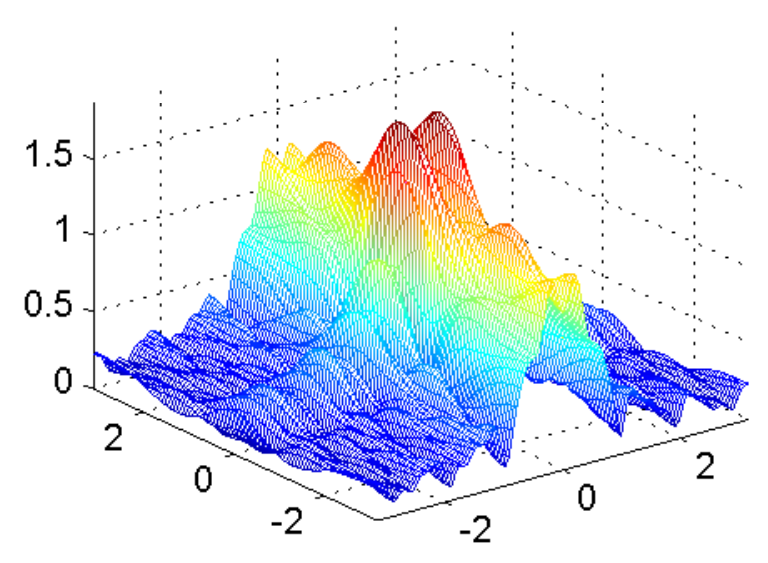




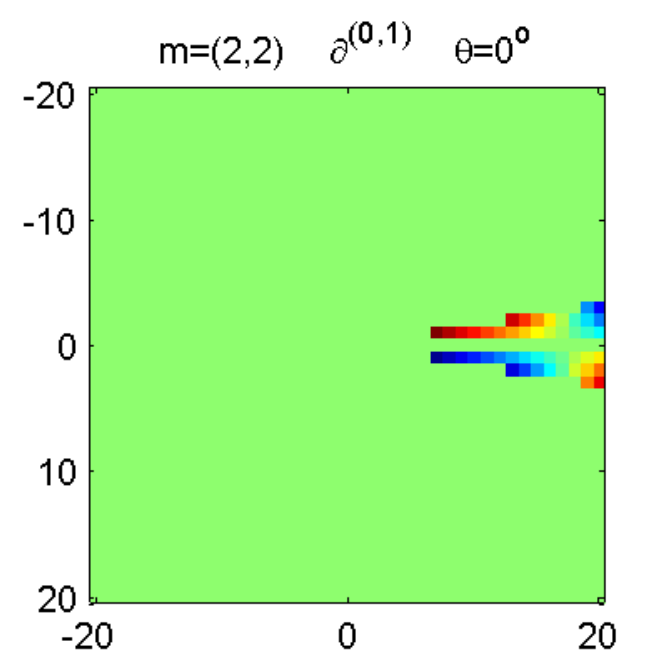

|FT|

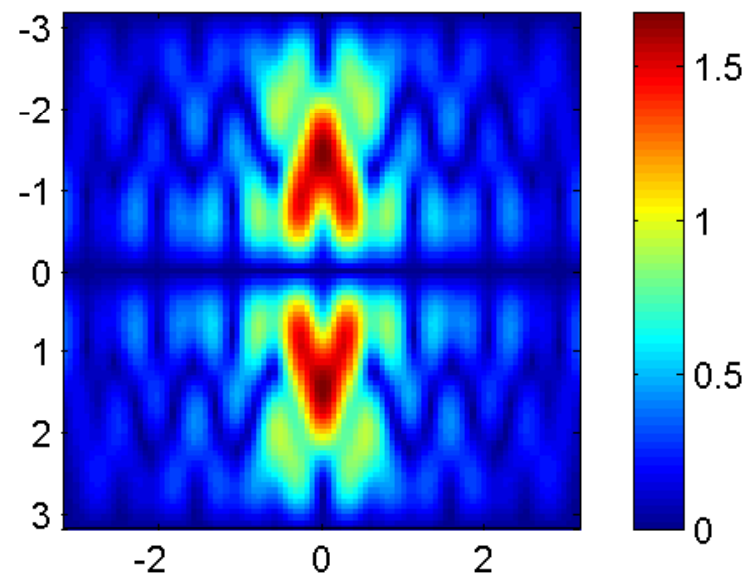

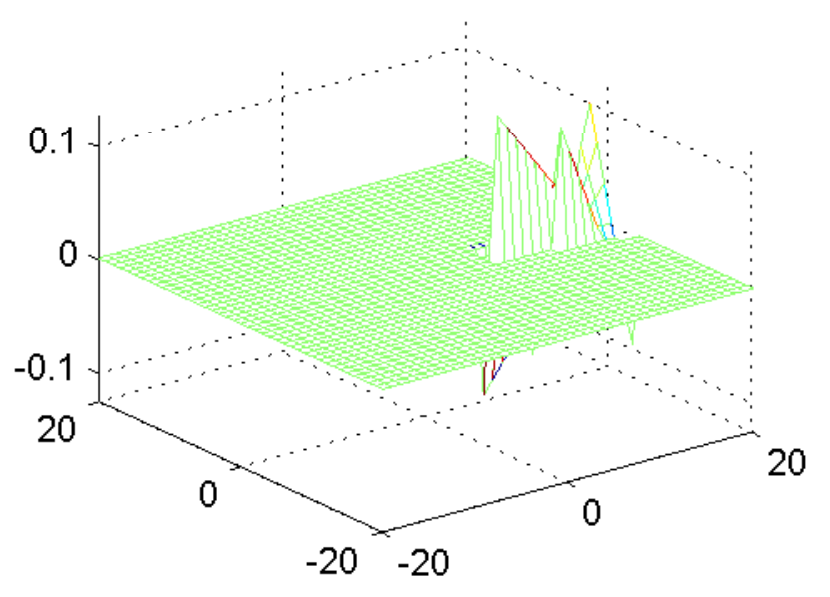

|FT|

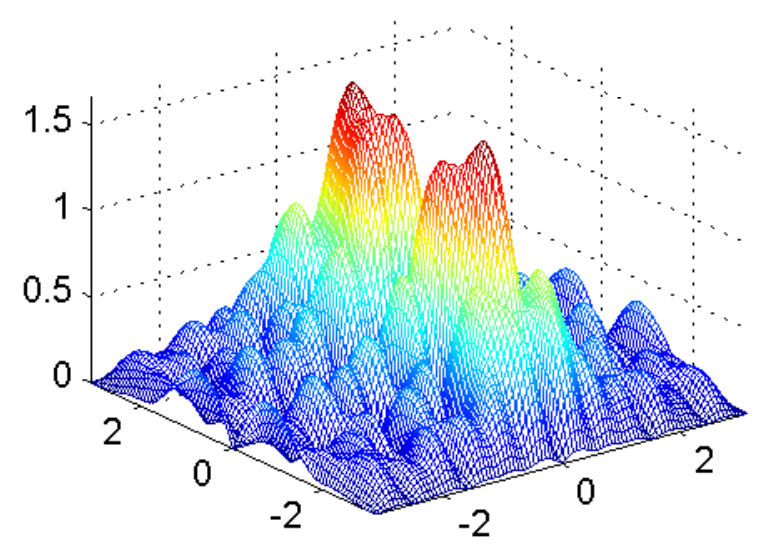



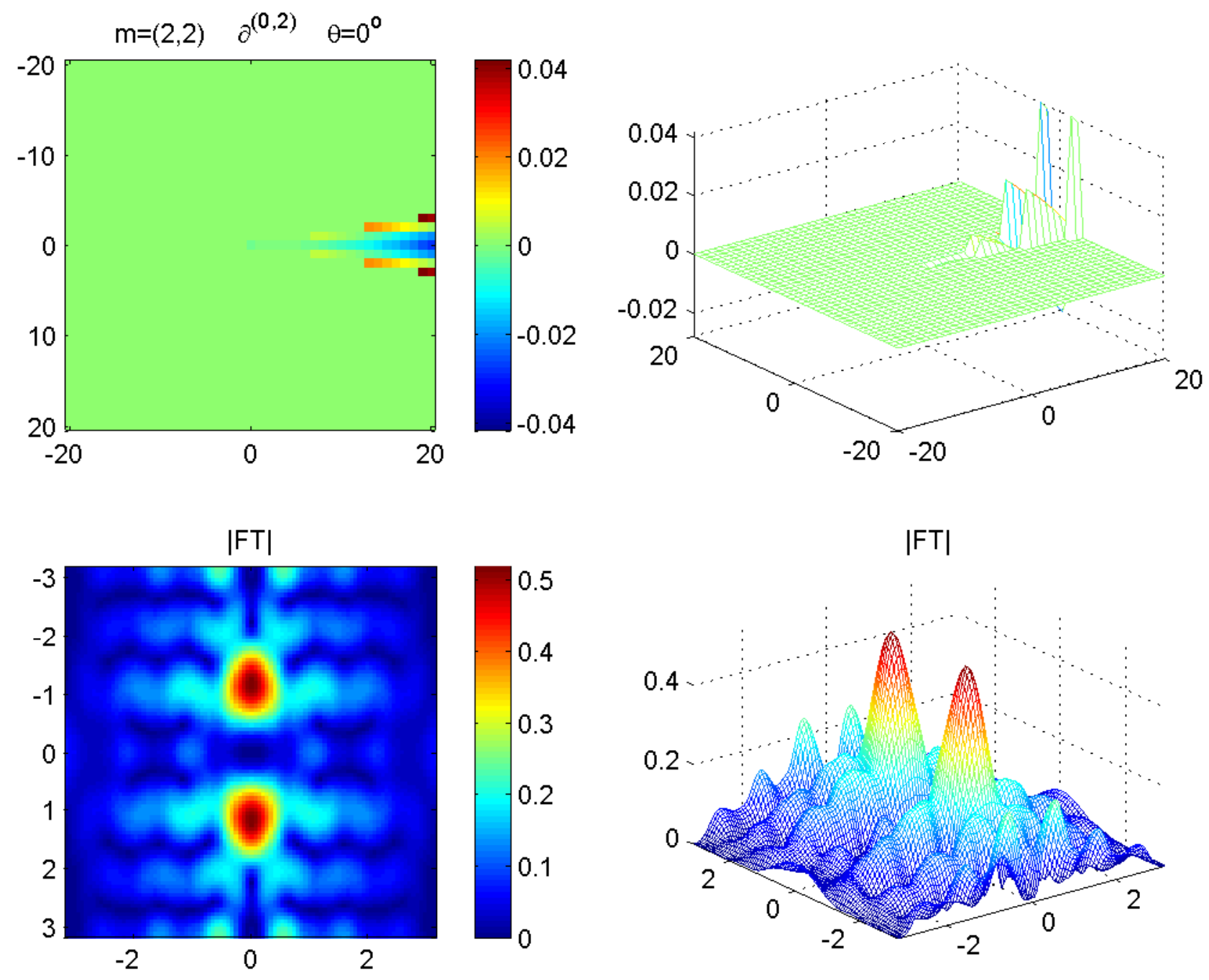

|FT|

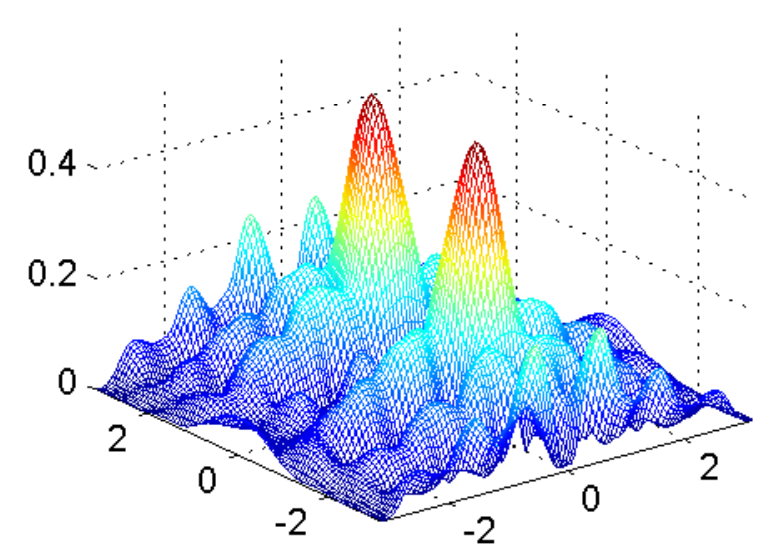



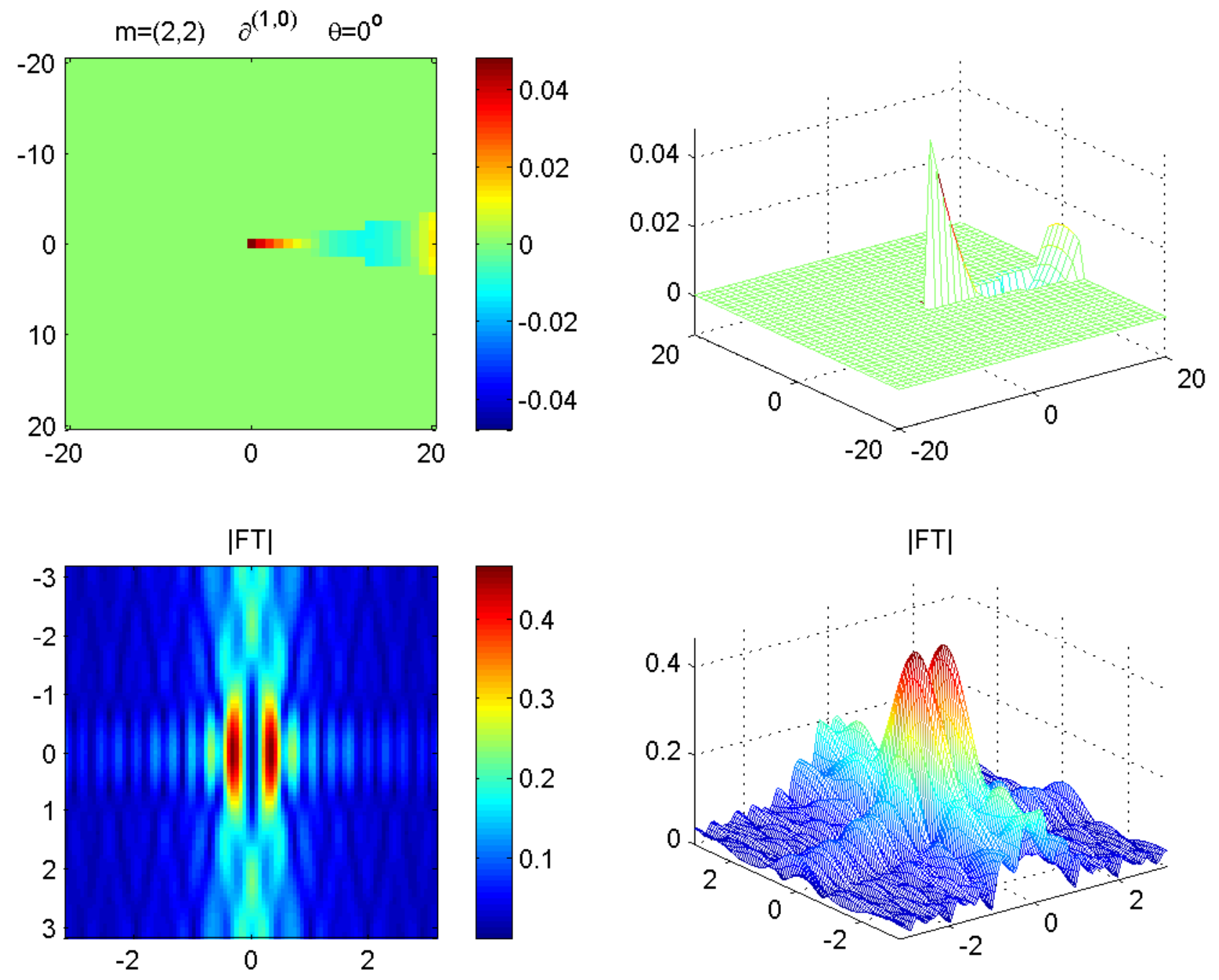

|FT|

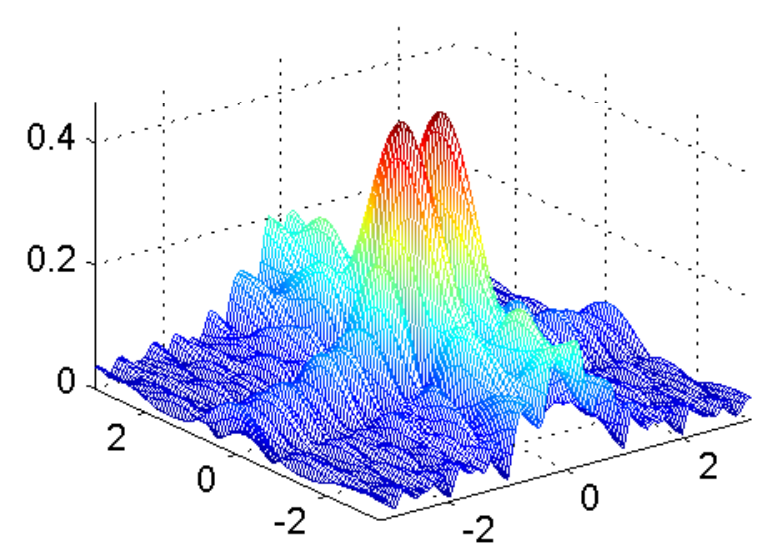



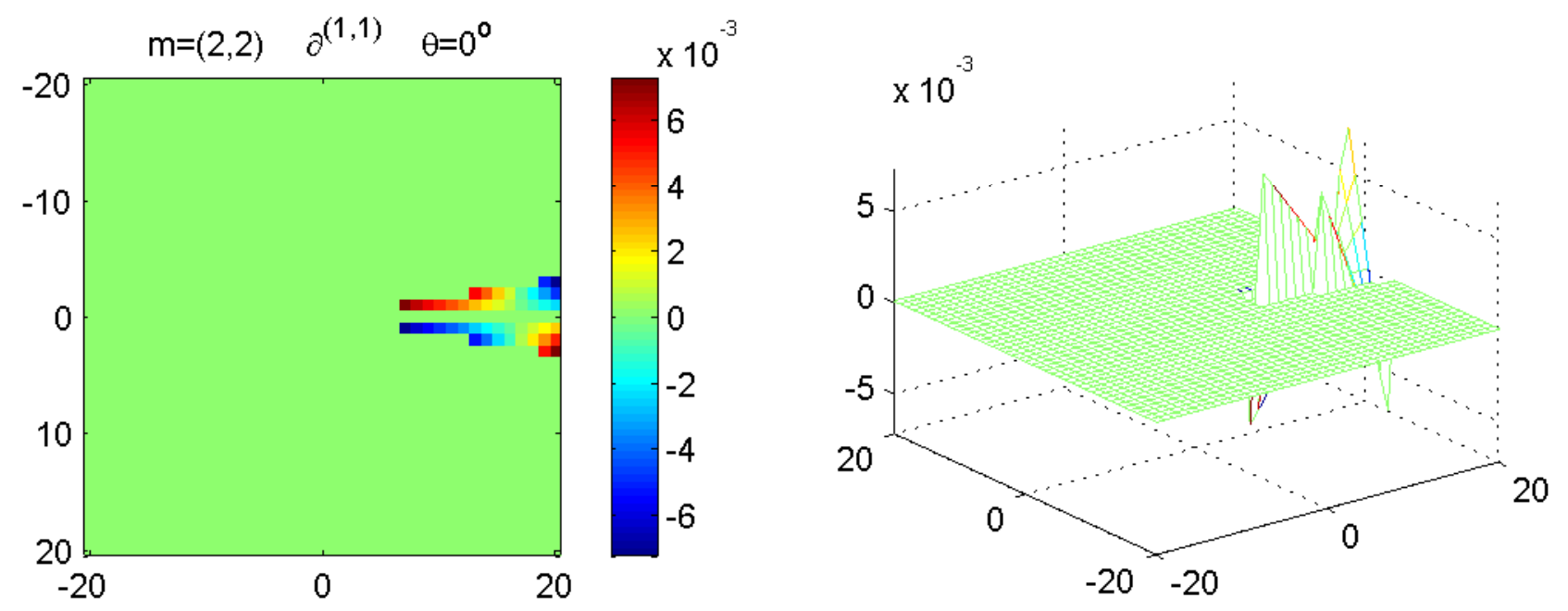

|FT|

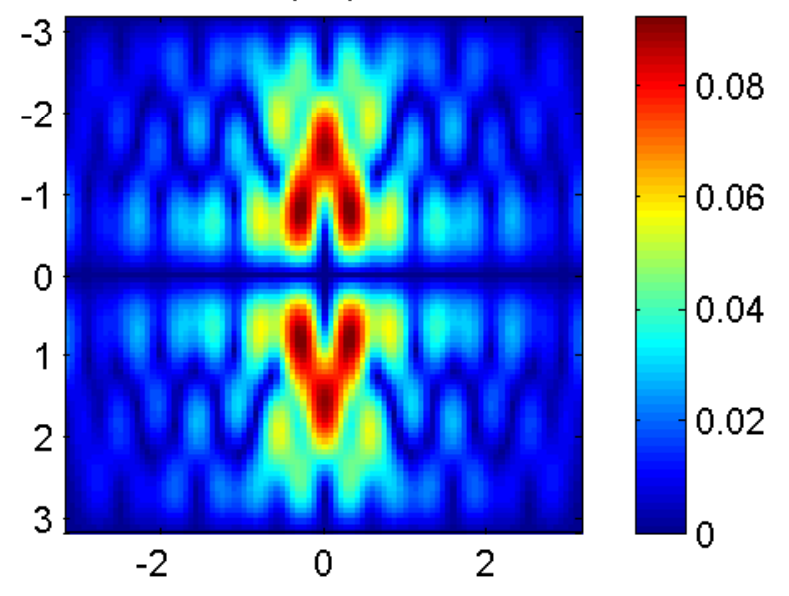

|FT|

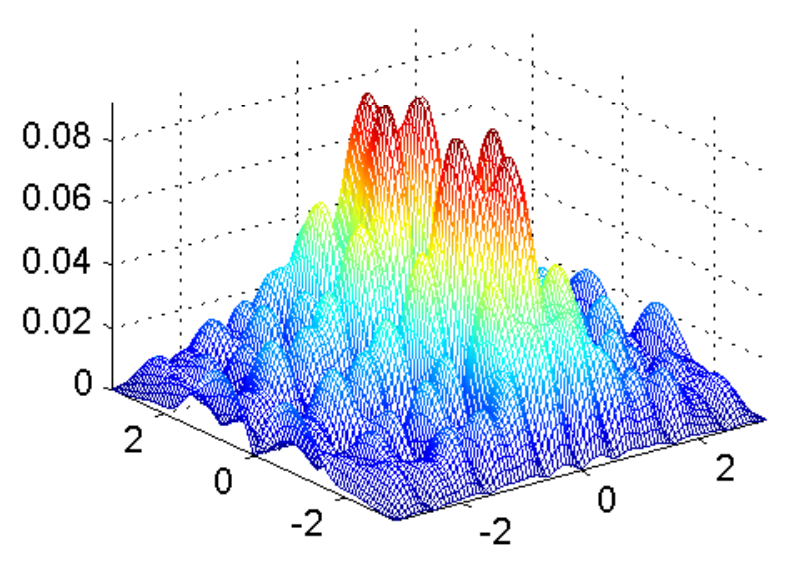



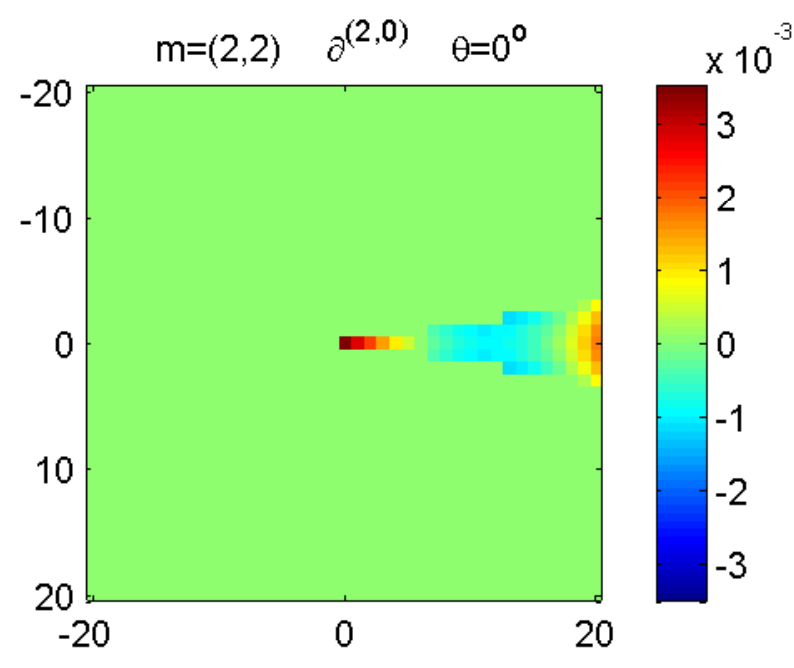

|FT|

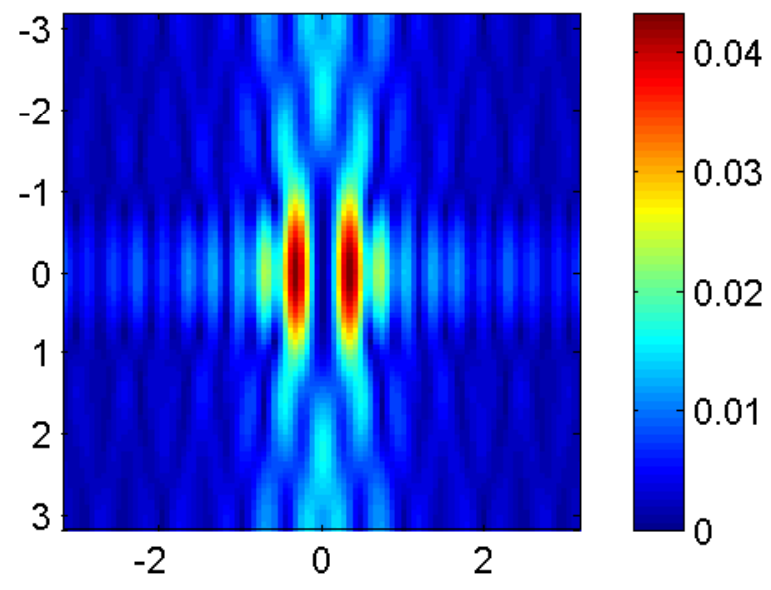

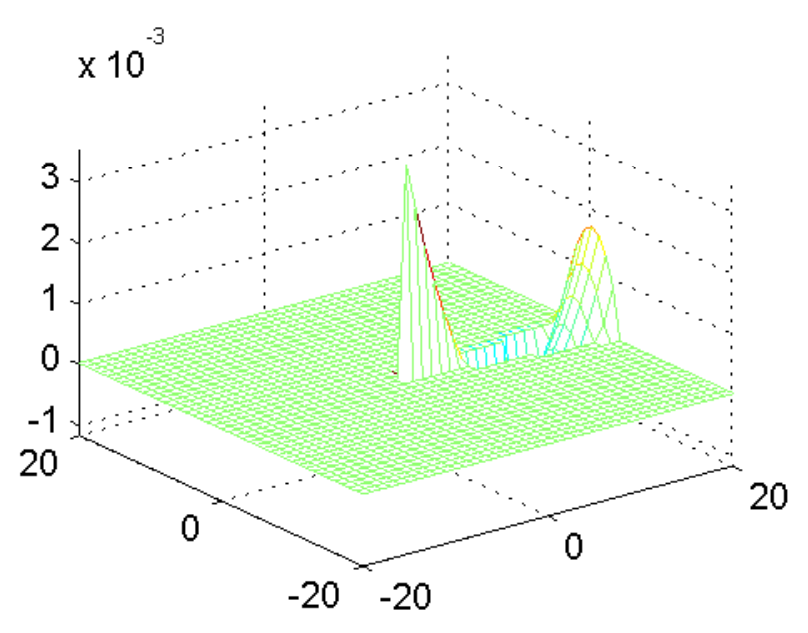

$|\mathrm{FT}|$

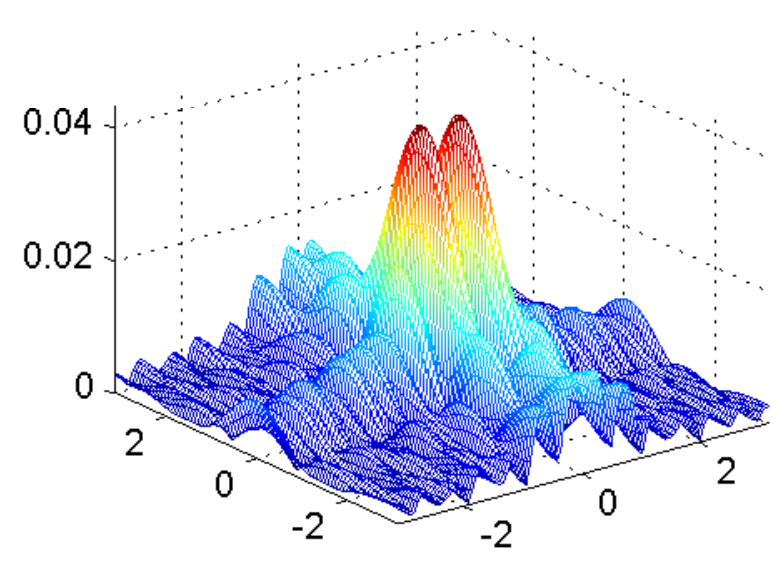




\section{(2) ICI rule}

Filtering of $h^{+}(x)$.

(3) Fusing of partial estimates

Let's $S$ partial estimates be obtained:

$$
\hat{y}_{h}^{[s]}(x), s=1, \ldots, S .
$$

There are a number of ways how to fuse these estimates. In particular:

$$
\hat{y}(x)=\sum_{k=1}^{S} \lambda_{s} \hat{y}_{h_{s}^{+}(x)}^{[s]}(x), \lambda_{s}=\frac{\operatorname{var}}{\operatorname{var}_{s}}, \operatorname{var}=1 / \sum_{s=1}^{S}\left(1 / \operatorname{var}_{s}\right),
$$

where $\hat{y}_{h_{s}^{+}(x)}^{[s]}(x)$ are the kernel estimates with the ICI rule adaptive window size for the subareas $s=1, \ldots, S$. 


\section{Algorithm complexity}

The calculation of the image estimate $\hat{y}_{h}^{[s]}$ for given $h$ is a linear

convolution requiring $N_{\text {conv }} \sim n \log n$ operations $n=n_{1} n_{2}$.

If the sectioning procedure is used for convolution, then

$N_{\text {conv }} \sim n \log n_{h_{J}}$, where $n_{h_{J}}$ is a maximum size of the square mask of the kernel $g_{h_{J}}$.

The selection of the adaptive scale the ICI algorithm is implemented as a loop on $J$ different scales in the set $H$.

These calculations are repeated for each of the $S$ subareas (quadrants) of the pixel neighborhood with the fusing the estimates.

Thus, overall the algorithm complexity is proportional to

$$
J \cdot S \cdot N_{\text {conv }},
$$

where $S$ is a number of the directional estimates. 


\section{Experiments}

The test signals are the $256 \times 256$ "Cameraman" image ( 8 bit gray-scale) and binary "Cheese" corrupted by an additive zero-mean Gaussian noise.

The blurred $S N R(B S N R)$ is defined in $d B$ as

$$
B S N R=10 \log _{10}\left[\sum_{x}\{(y \circledast v)(x)-\operatorname{mean}((y \circledast v)(x))\}^{2} / \sigma^{2} n_{1} n_{2}\right],
$$

with $B S N R=40 \mathrm{~dB}$.

The discrete-space blur convolution $P S F$ is a uniform $9 \times 9$ box-car. The $L P A$ is defined with $H=\{1,3,5,9,17\}$. 
The performance criteria:

(1) Root mean squared error $(R M S E): R M S E=\sqrt{\frac{1}{\#} \sum_{x}(y(x)-\hat{y}(x))^{2}}$;

(2) Improvement in SNR (ISNR) in $d B$ :

$$
I S N R=20 \log _{10}\left(\sqrt{\frac{1}{\#} \sum_{x}(y(x)-z(x))^{2}} / R M S E\right) .
$$

(3) Visual evaluation.

Mainly the comparison is produced with one of the best in the field wavelet based algorithm developed in RICE University (Neelamani R., Choi H., Baraniuk R.). It is called "ForWaRD" and available from http://www.dsp.rice.edu/software/. 


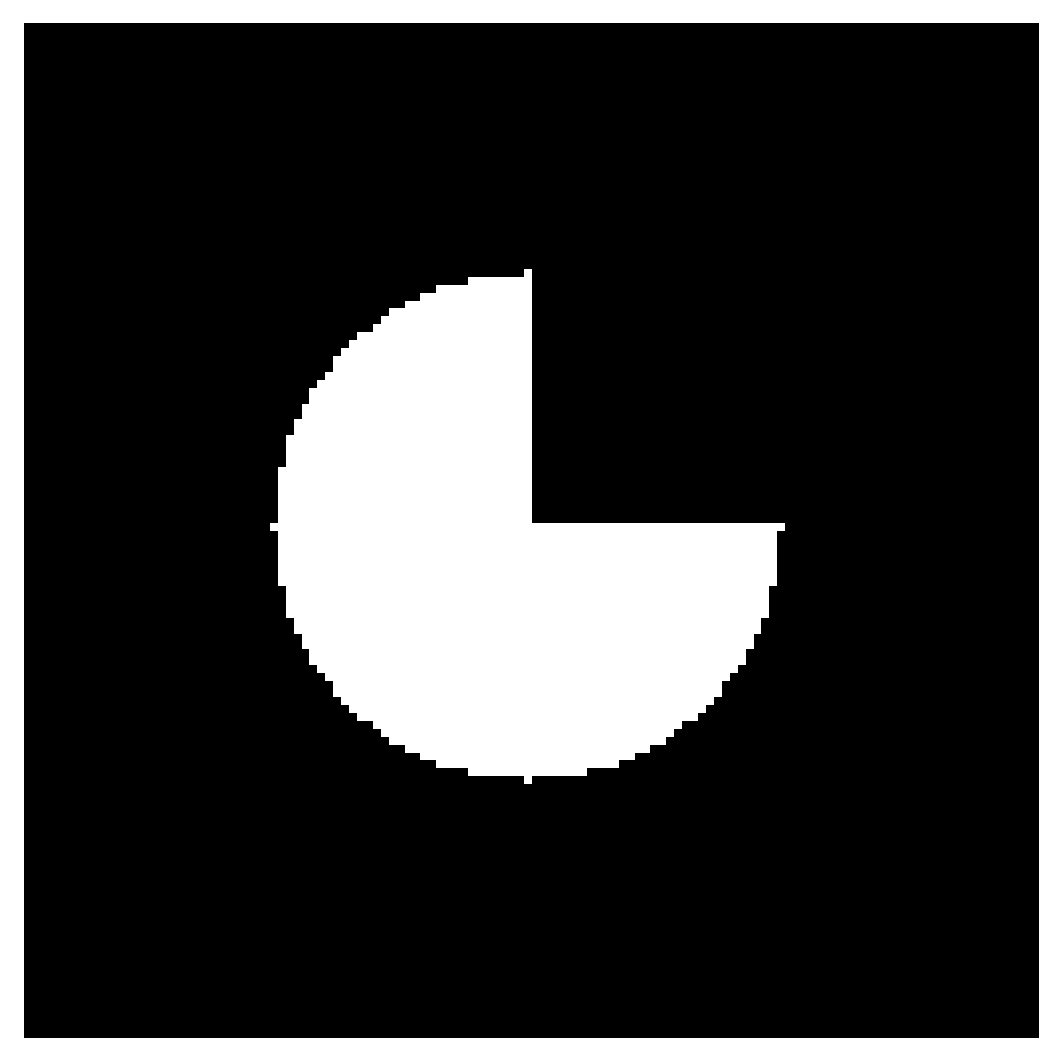

"Cheese" original

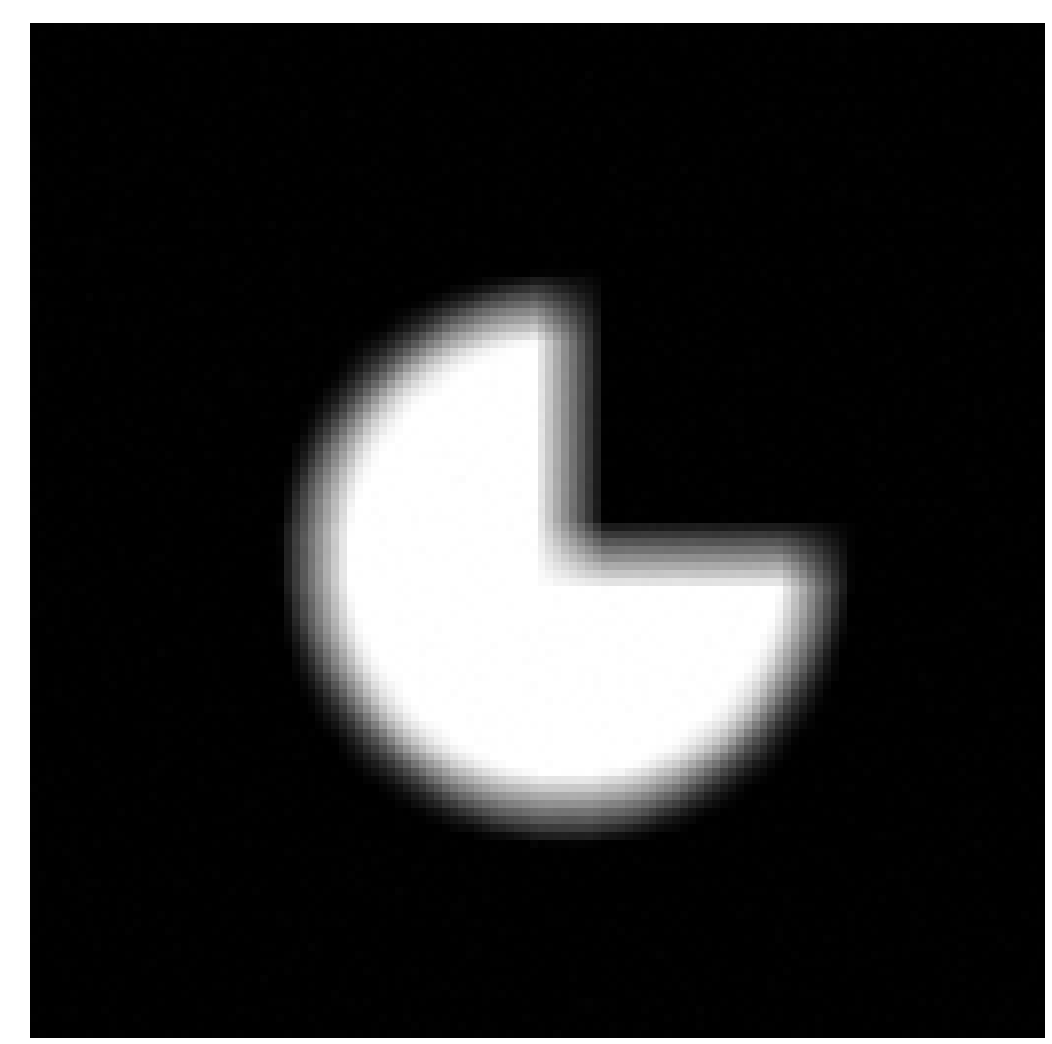

"Cheese" observations 


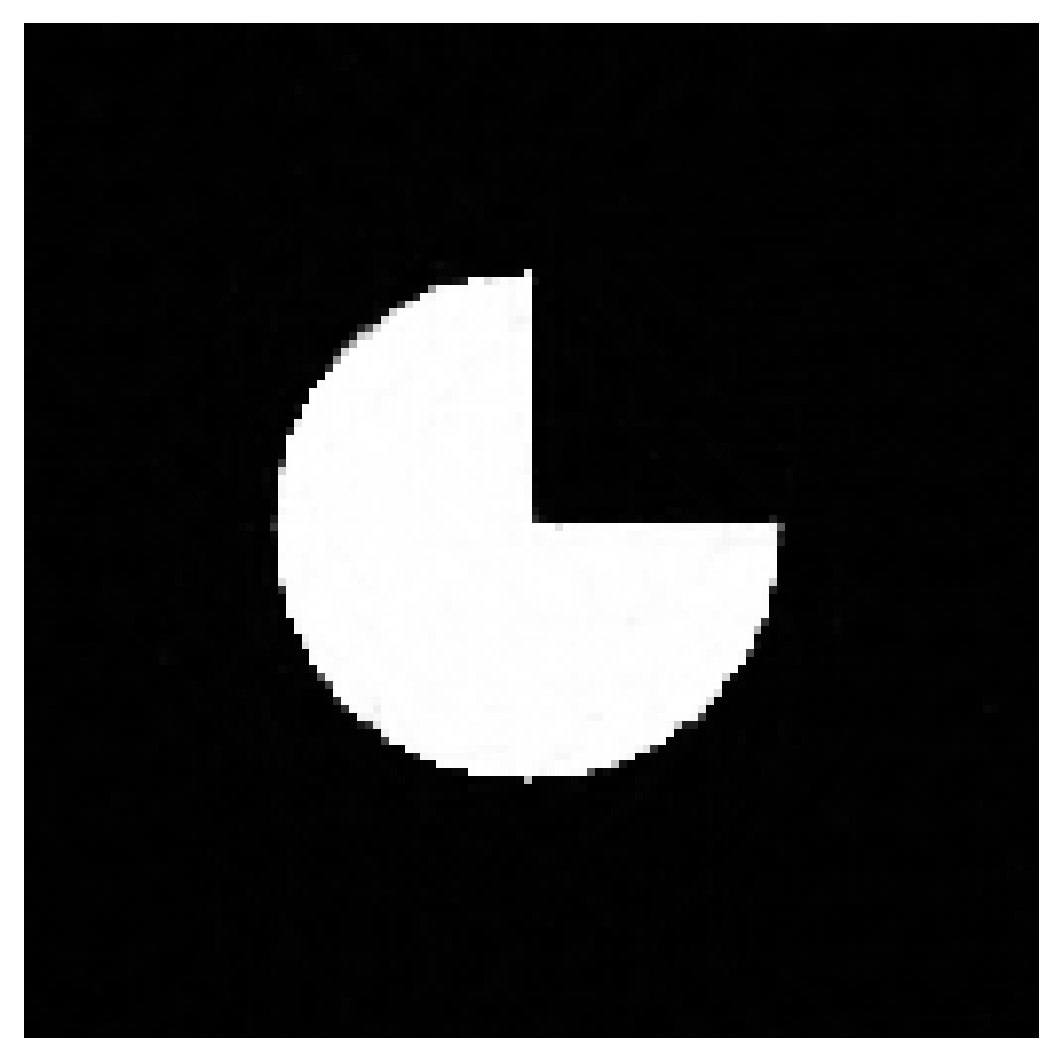

$L P A-I C I, I S N R=15.8 d B$

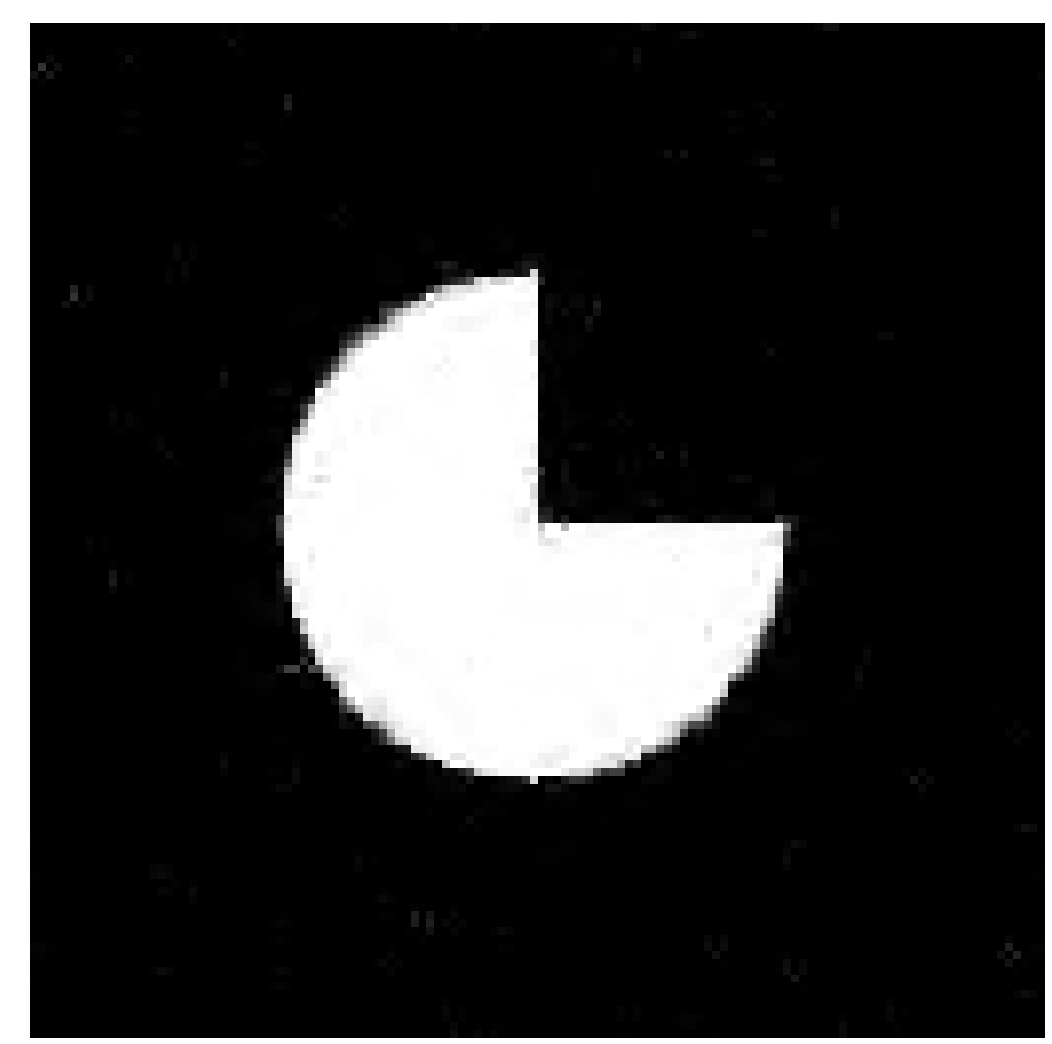

ForWard, $I S N R=9.55 \mathrm{~dB}$ 

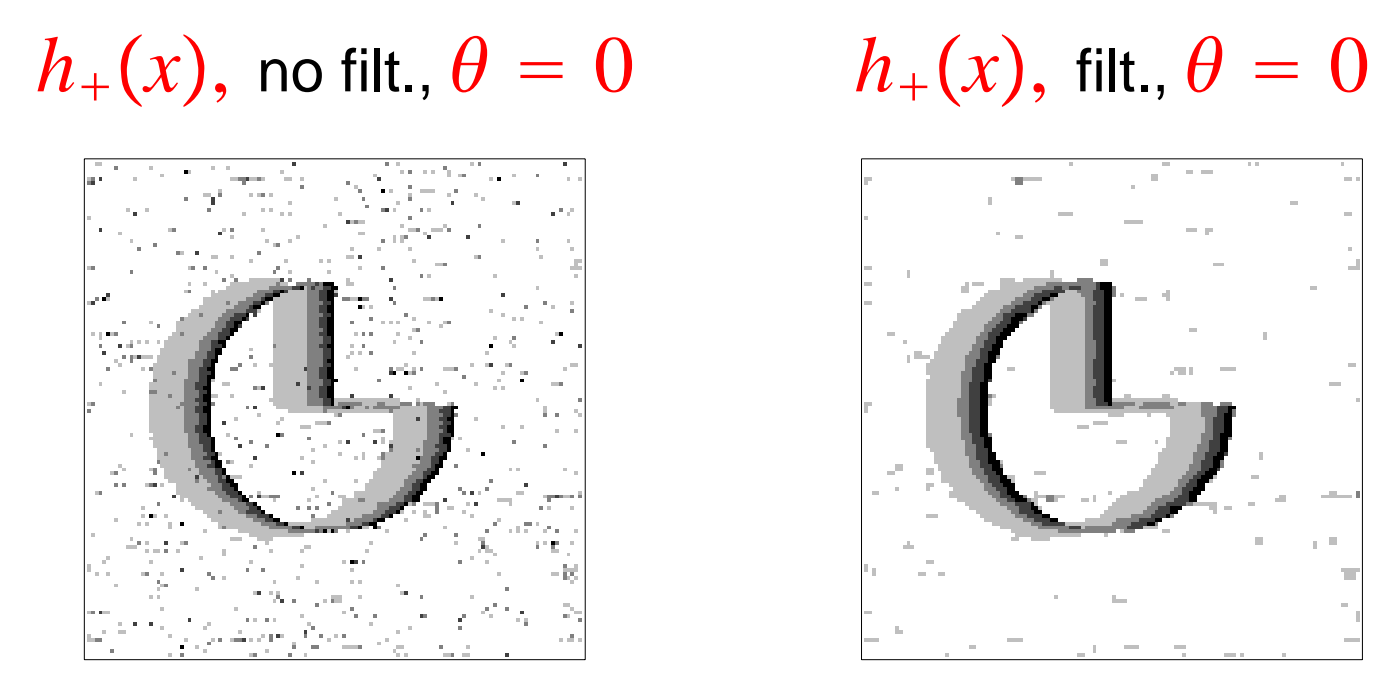

$h_{+}(x)$, no filt., $\theta=45^{0}$

$h_{+}(x)$, filt., $\theta=45^{0}$
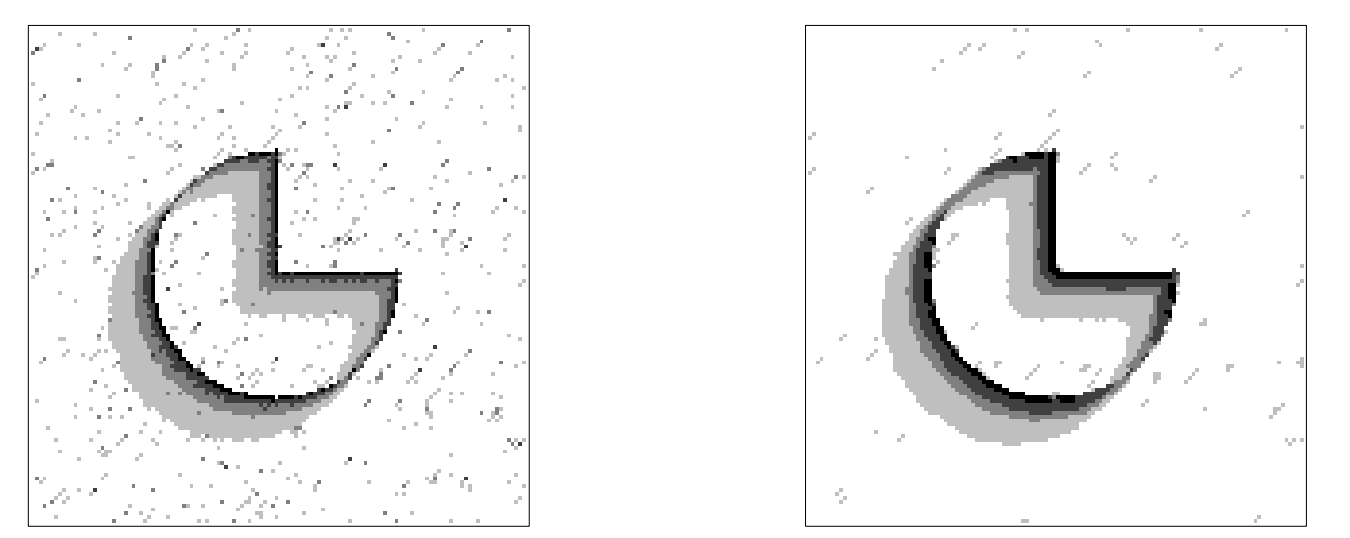


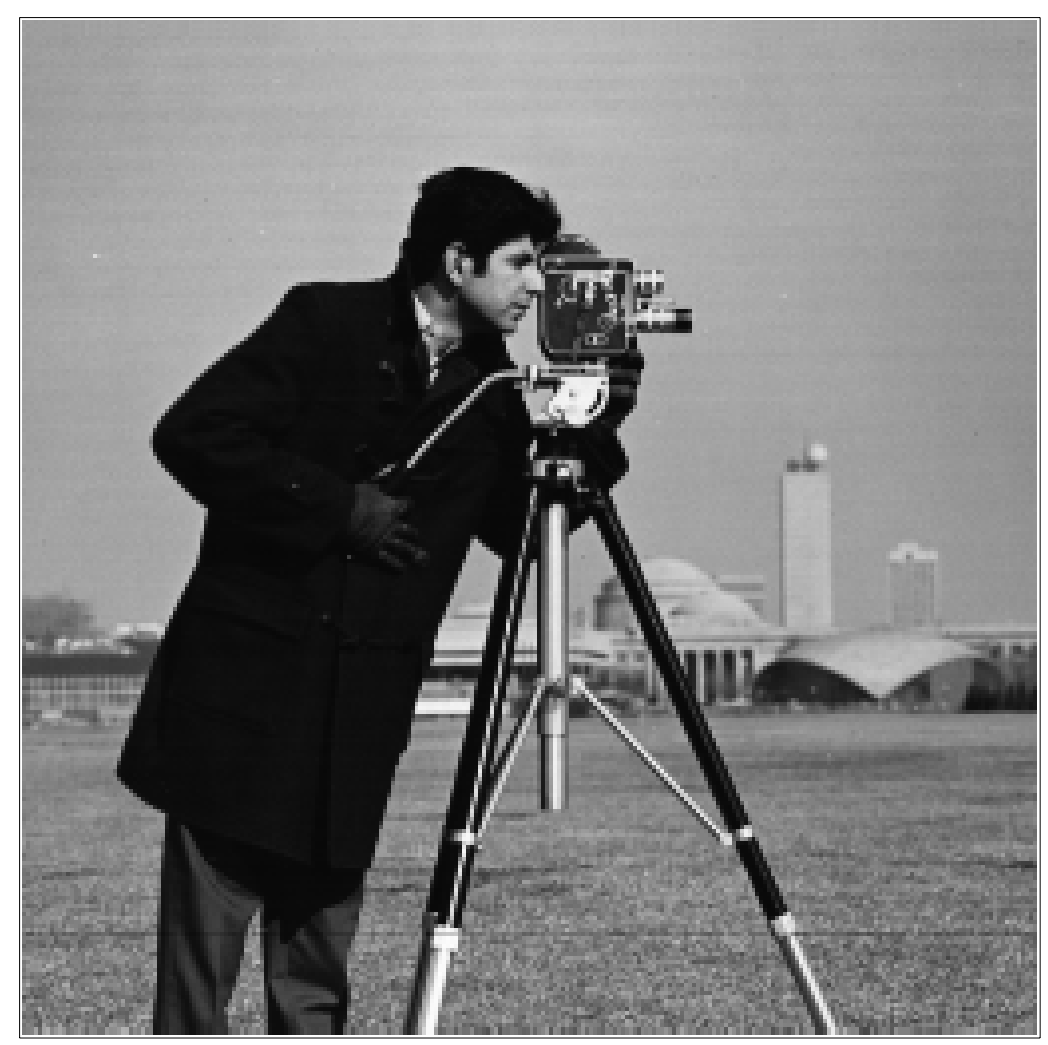

Original

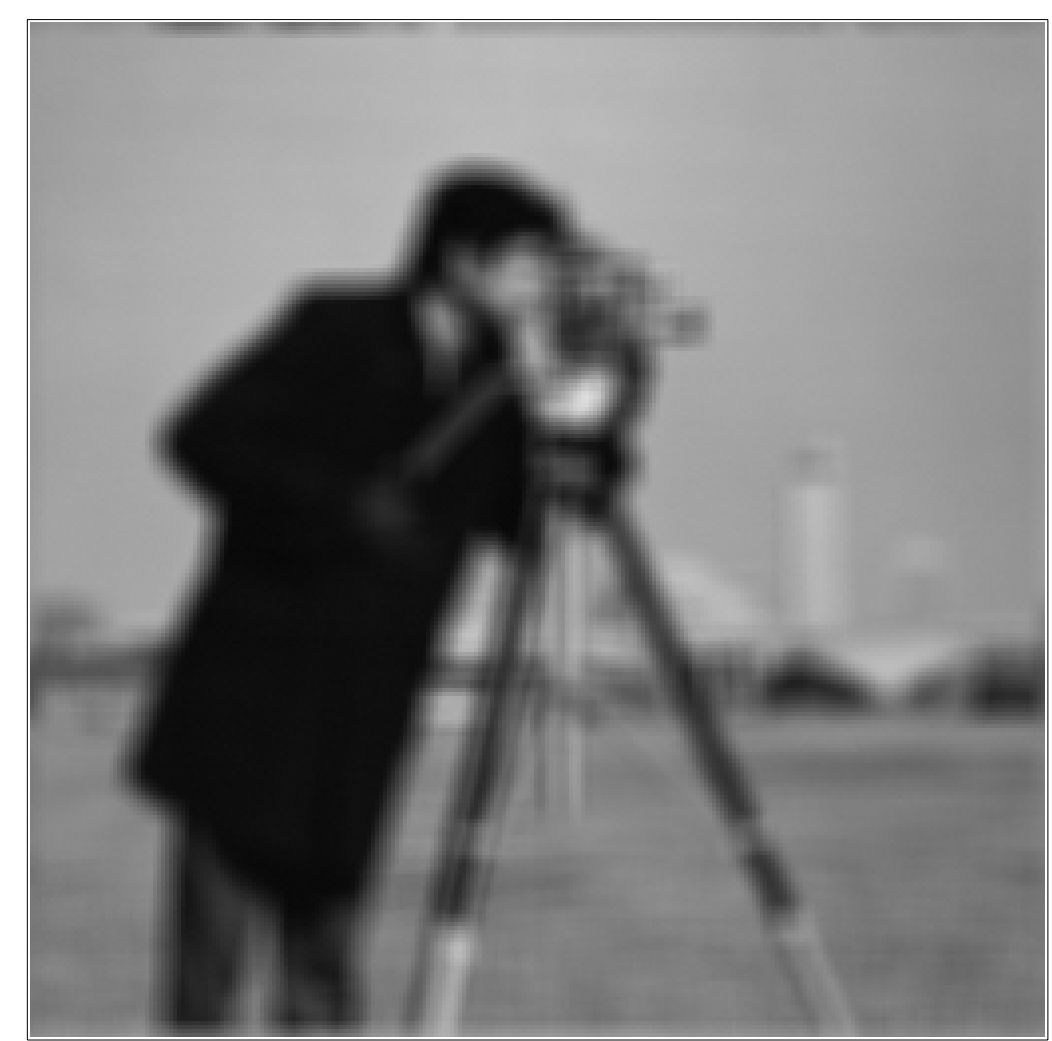

Observations 


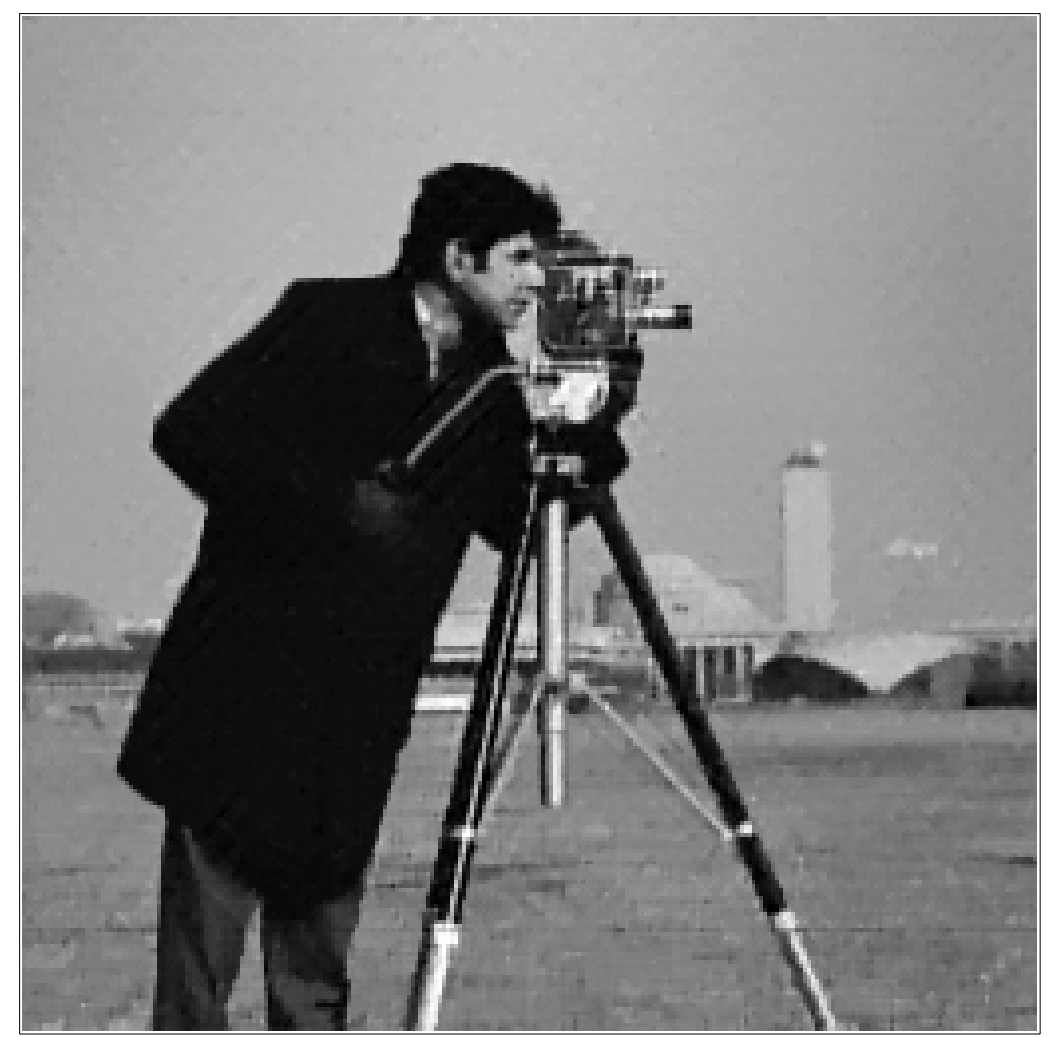

$L P A-I C I, I S N R=8.23 d B$

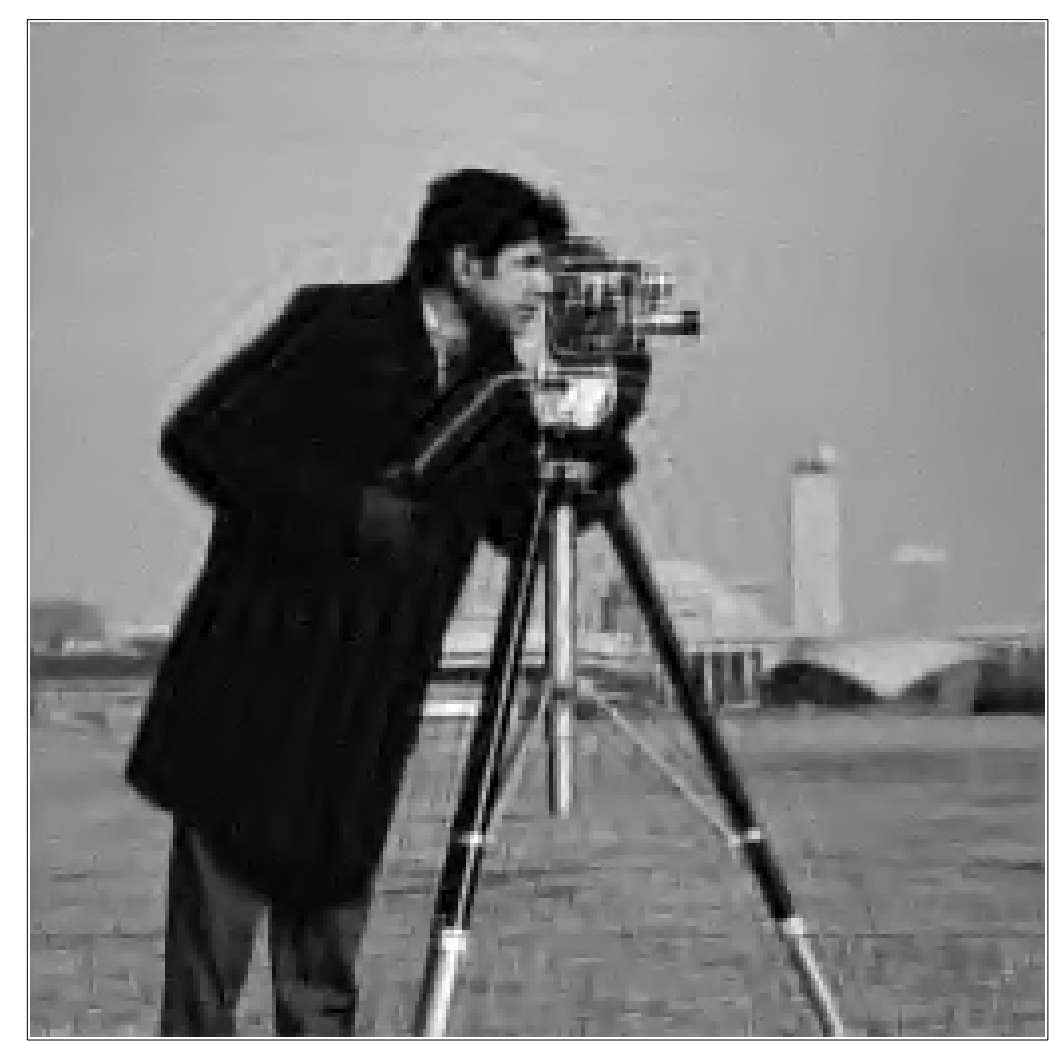

ForWard, ISNR $=7.16 \mathrm{~dB}$ 


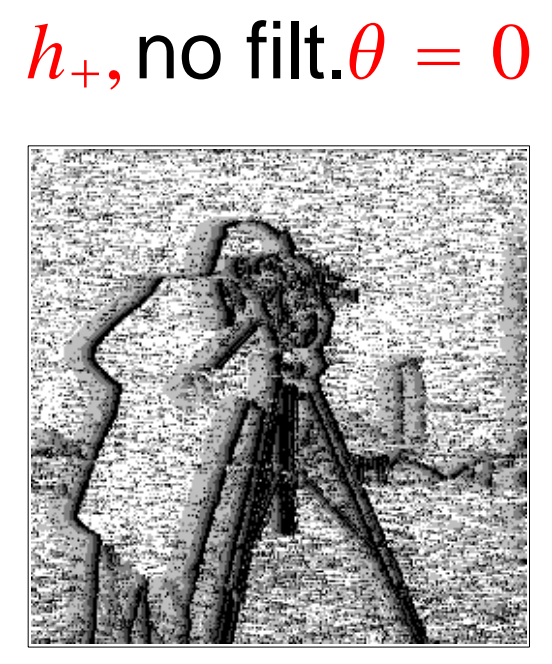

$h_{+}$, no filt. $\theta=45^{0}$

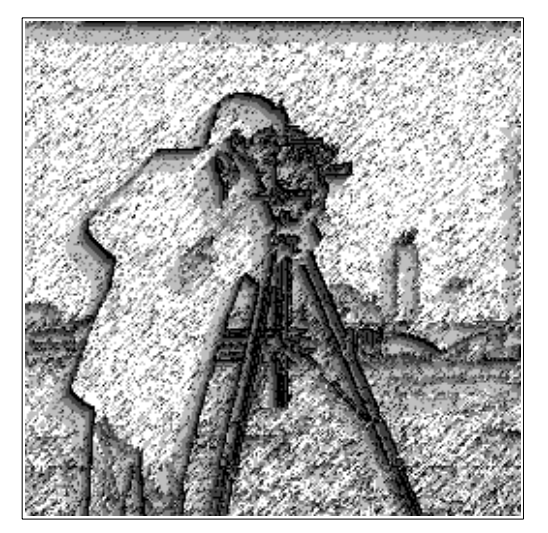

$h_{+}$, filt. $\theta=0$

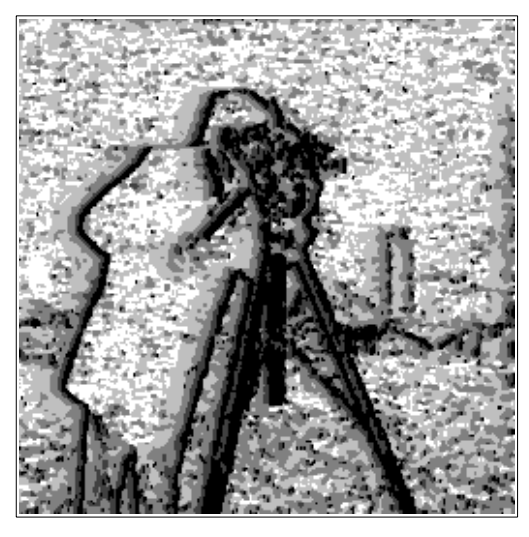

$h_{+}$, filt. $\theta=45^{0}$

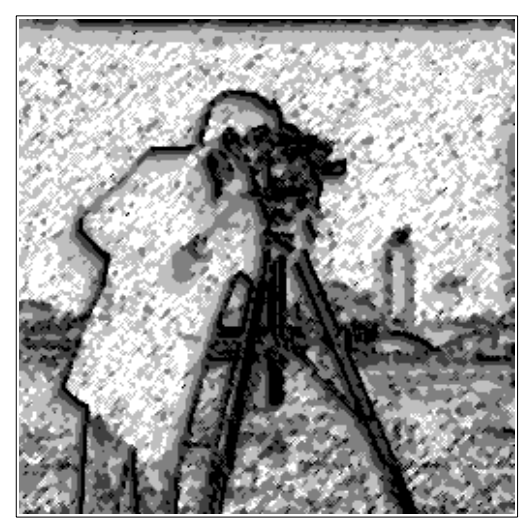


TABLE I : "Cameraman", $9 \times 9$ car-box PSF

\begin{tabular}{|l|l|}
\hline Method & ISNR \\
\hline LPA $-I C I$ & $8.23 \mathrm{~dB}$ \\
\hline Result by Figueiredo and Nowak (EM) & $7.59 \mathrm{~dB}$ \\
\hline Forward, & $7.16 \mathrm{~dB}$ \\
\hline Result by Banham and Katsaggelos & $6.7 \mathrm{~dB}$ \\
\hline
\end{tabular}


TABLE II : "Lena", PSF is a $5 \times 5$ separable filter with the weights $[1,4,6,4,1] / 16$ in horizontal and vertical directions, $B S N R=15.93 \mathrm{~dB}$.

\begin{tabular}{|l|l|}
\hline Method & ISNR \\
\hline LPA $-I C I$ & $3.76 \mathrm{~dB}$ \\
\hline Best result by Figueiredo and Nowak (EM) & $2.94 \mathrm{~dB}$ \\
\hline Best result by Liu and Moulin & $1.078 \mathrm{~dB}$ \\
\hline ForWaRD & $2.87 \mathrm{~dB}$ \\
\hline
\end{tabular}




\section{Conclusions}

Some theoretical topics:

(1) Directional nonparametric regression estimation;

(2) The adaptive scale selection procedures for small number of samples;

(3) Fusing of the partial directional estimates;

(4) Analysis of regularized and Wiener based algorithms. 


\section{Asymptotic properties}

Oracle accuracy:

(B1) $y \in H_{r}$

$$
H_{r}=\left\{\max _{r_{1}+r_{2}=r}\left|\partial^{r_{1}+r_{2}} y(x) / \partial x_{1}^{r_{1}} \partial x_{2}^{r_{2}}\right|=L_{r}(x) \leq \bar{L}_{r}, \forall r_{1}+r_{2}=r\right\} .
$$

(B2) $V(\lambda)$ is polynomial decaying,

$$
\bar{c}_{0} \geq\left|V ( \lambda ) \left\|\left|\lambda\left\|^{\alpha} \geq c_{0}, \forall\right\| \lambda \|>A, \min _{\|\lambda\| \leq A}\right| V(\lambda) \mid=c_{1} .\right.\right.
$$

Then, as $h, \Delta, \Delta / h \rightarrow 0, m \leq r$,

$$
r\left(x, h^{*}(x)\right)=O\left(n^{-\frac{2(m+1)}{m+\alpha+2}}\right) .
$$

Adaptive estimate accuracy:

$$
r\left(x, h^{+}(x)\right)=O\left((\ln n / n)^{\frac{2(m+1)}{m+\alpha+2}}\right) .
$$

can be proved based on the technique due to A.Goldenshluger, A. 
Nemirovski (1997) for $1 D$ regression and A. Goldenshluger (1999) for $1 D$ continuous deconvolution. 


\section{References}

Banham M. R. and A.K. Katsaggelos, "Spatially adaptive wavelet-based multiscale image restoration," IEEE Trans. Image Processing, vol. 5, pp. 619-634, 1996.

A. Goldenshluger, "On pointwise adaptive nonparametric deconvolution," Bernoulli, vol. 5, pp. 907-925, 1999.

A. Goldenshluger and A. Nemirovski, "On spatial adaptive estimation of nonparametric regression," Math. Meth. Statistics vol. 6, pp. 135-170, 1997.

V. Katkovnik, Nonparametric identification and smoothing of data (Local approximation methods). Nauka, Moscow, 1985 (in Russian).

V. Katkovnik, "A new method for varying adaptive bandwidth selection," IEEE Trans. on Signal Processing, vol. 47, N²9, pp. 2567-2571, 1999.

V. Katkovnik, K. Egiazarian, and Jaakko Astola, "Adaptive window size image de-noising based on intersection of confidence intervals ( $\mathrm{ICl}$ ) rule," Journal of Mathematical Imaging and Vision, vol. 16, N 3. pp. 223-235, 2002.

V. Katkovnik, K. Egiazarian, and Jaakko Astola, Adaptive varying scale methods in image 
processing. Tampere International Center for Signal Processing, TICSP Series, N 19, Tampere, TTY, Monistamo, 2003.

V. Katkovnik, K. Egiazarian, and Jaakko Astola, "A spatially adaptive nonparametric regression image deblurring," IEEE Trans Image Processing, submitted, 2003.

Katkovnik, V., "A multiresolution nonparametric regression and image de-nosing," Proceedings of ICIP 2003, Barcelona, Spain, 14-17 September, 2003.

V. Katkovnik, K. Egiazarian, and Jaakko Astola, "Spatially adaptive deblurring based on nonparametric regression ," ICIP 2004, Singapore, submitted, 2004.

V. Katkovnik, A. Foi, K. Egiazarian, and J. Astola, "Directional varying scale approximations for anisotropic signal processing", EUSIPCO 2004, Wien, submitted, 2004.

R. Neelamani, H. Choi, and R. G. Baraniuk, "Forward: Fourier-wavelet regularized deconvolution for ill-conditioned systems," IEEE Trans. on Image Processing, 2003.

Figueiredo M. A.T. and R. D. Nowak," An EM algorithm for wavelet-based image restoration," IEEE Trans Image Processing, vol. 12, no. 8, pp. 906-916, 2003. 